\title{
WIPI1 is a conserved mediator of right ventricular failure
}

\author{
Christos Tzimas, ${ }^{1}$ Christoph D. Rau, ${ }^{2}$ Petra E. Buergisser, ${ }^{1}$ Gaston Jean-Louis Jr., ${ }^{1}$ Katherine Lee, ${ }^{1,3}$ \\ Jeffrey Chukwuneke, ${ }^{1}$ Wen Dun, ${ }^{1}$ Yibin Wang, ${ }^{2}$ and Emily J. Tsai ${ }^{1,4}$ \\ 'Division of Cardiology, Department of Medicine, Columbia University Vagelos College of Physicians and Surgeons, New \\ York, New York, USA. 'Division of Molecular Medicine, Department of Anesthesiology and Perioperative Medicine, David \\ Geffen School of Medicine, UCLA, Los Angeles, California, USA. Institute of Human Nutrition, Columbia University \\ Vagelos College of Physicians and Surgeons, New York, New York, USA. ${ }^{4}$ Cardiovascular Research Center, Lewis Katz School \\ of Medicine, Temple University, Philadelphia, Pennsylvania, USA.
}

Right ventricular (RV) dysfunction is highly prevalent across cardiopulmonary diseases and independently predicts death in both heart failure (HF) and pulmonary hypertension (PH). Progression towards RV failure (RVF) can occur in spite of optimal medical treatment of HF or PH, highlighting current insufficient understanding of RVF molecular pathophysiology. To identify molecular mechanisms that may distinctly underlie RVF, we investigated the cardiac ventricular transcriptome of advanced-HF patients, with and without RVF. Using an integrated systems genomic and functional biology approach, we identified an RVF-specific gene module, for which WIPI1 served as a hub and HSPB6 and MAP4 as drivers, and confirmed the ventricular specificity of Wipi1, Hspb6, and Map4 transcriptional changes in adult murine models of pressure overloadinduced RV versus left ventricular failure. We uncovered a shift towards noncanonical autophagy in the failing RV that correlated with RV-specific Wipi1 upregulation. In vitro siRNA silencing of Wipi1 in neonatal rat ventricular myocytes limited noncanonical autophagy and blunted aldosteroneinduced mitochondrial superoxide levels. Our findings suggest that Wipi1 regulates mitochondrial oxidative signaling and noncanonical autophagy in cardiac myocytes. Together with our human transcriptomic analysis and corroborating studies in an RVF mouse model, these data render Wipi1 a potential target for RV-directed HF therapy.

Conflict of interest: EJT and CT are co-inventors on a provisional patent application $(62 / 836,315)$ that was filed on April 19, 2019 with the US Patent and Trademark Office. The patent relates to the pharmacologic treatment of right ventricular failure.

Copyright: (c) 2019 American Society for Clinical Investigation

Submitted: July 5, 2018

Accepted: April 23, 2019

Published: June 6, 2019.

Reference information: JCl Insight. 2019;4(11):e122929. https://doi. org/10.1172/jci.insight.122929.

\section{Introduction}

Right ventricular dysfunction (RVD) predicts worse clinical outcomes, including death, in patients with heart failure (HF) (1-6) and pulmonary hypertension (PH) (7-9), irrespective of etiology, left ventricular (LV) function, or pulmonary artery pressures. Although RVD is largely a consequence of $\mathrm{PH}$, left heart disease is the most common etiology of $\mathrm{PH}$, and HF patients with RVD outnumber other $\mathrm{PH}$ patients with RVD. More than half of all HF patients are estimated to have RVD: $25 \%-50 \%$ of HF patients with preserved LV ejection fraction (HFpEF) and up to $75 \%$ of those with reduced LV ejection fraction (HFrEF) (5, 10, 11). Strikingly, current guideline-directed HF pharmacotherapies neither reverse RVD nor prevent RV failure (RVF) (12). This discrepancy should not be surprising. The RV is distinct from the LV with regards to embryological origin, morphology, physiology, and response to stress. Diuretics, inotropic medications, and, in select cases, advanced pulmonary vasodilators are empiric medications used for managing RVF. However, none of these improve long-term outcomes in HF patients, and there remain no RV-targeted therapies for either $\mathrm{PH}$ or $\mathrm{HF}$ patients.

To date, efforts in drug development for $\mathrm{PH}$ and $\mathrm{HF}$ have focused, respectively, on pulmonary vascular remodeling and LV function, not RV function directly. Preclinical studies have been challenged by limitations of commonly used animal models that were developed to study pulmonary vascular disease rather than RV dysfunction or biventricular HF. Some studies have sought to identify molecular signatures of RVF, using animal models (13-17) or human tissue (18-20). Most of these were limited to animal models or patients with RV dysfunction but not $\operatorname{RVF}(13,14,16,18,19)$. These prior studies did not pursue experimental validation or mechanistic studies of their potential molecular signatures of RV dysfunction (13-20). All relied on biased pathway analyses of select differentially expressed genes. Although widely used, pathway analyses are limited 
by an emphasis on individual genes, the resolution of the associated knowledge base, potentially incorrect and inaccurate annotations, and lack of dynamic information (21).

Here, we sought to analyze the ventricular transcriptome of advanced-HF patients, with versus without hemodynamically significant RVF, to identify gene networks that may be uniquely altered in RVF. We integrated weighted gene coexpression network analysis (WGCNA) with detailed hemodynamic indices of advanced-HF patients to identify a gene network (module) that correlated specifically with RVF. By validating gene hubs and drivers of this network in murine HF models, we identified Wipil as a conserved mediator of RVF. Furthermore, silencing Wipil in aldosterone-stimulated, isolated neonatal rat cardiac myocytes blunted excessive noncanonical autophagy and mitochondrial superoxide levels, suggesting Wipil as a potential target for therapeutic intervention.

\section{Results}

Clinical and hemodynamic characteristics of advanced-HF patients. Clinical characteristics of advancedHFrEF patients without RVF and thereby with LV failure (LVF) alone, advanced-HFrEF patients with RVF and thereby biventricular failure (BiV-HF), and nonfailing (NF) adult patients are listed in Supplemental Table 1; supplemental material available online with this article; https://doi.org/10.1172/jci. insight.122929DS1. The median (interquartile range) age was $61.5(60.0-63.5)$ years for all advanced-HF patients and 51.0 (43.0-52.0) years for NF donors. There was no significant difference in age between LVF and BiV-HF patients. As would be expected, BiV-HF patients had higher rates of inotropic medication use, lower rates of beta blocker use, lower LVEF, and worse hemodynamic indices of RV function than LVF patients (Supplemental Tables 1 and 2). Specifically, BiV-HF patients had markedly elevated right atrial pressure (RA), increased ratio of RA to pulmonary capillary wedge pressure (RA/PCWP), lower systolic and mean arterial blood pressure (SBP and MAP), markedly decreased ratio of mean arterial pressure to RA (MAP/RA), and lower cardiac index (CI) in spite of greater inotropic support.

Transcriptomic analysis identifies a gene module uniquely associated with RVF. We used WGCNA to identify genetic pathways and groups of genes that distinguish the RV from the whole heart (22). Using only genes that were expressed (average FPKM $>1$ ) and variable (coefficient of variation $>10 \%$ across all cohorts) in the RV, we partitioned 13,613 transcripts into 23 RV-derived gene modules. Each module was defined by a tighter clustering coefficient compared with the network as a whole. We examined the correlation of the eigengene for each of the $23 \mathrm{RV}$-derived network modules with hemodynamic indices of RVF. Consequently, we identified one module that correlated significantly with elevated RA, elevated RA/PCWP, decreased SBP, and decreased CI (Supplemental Figure 1). This RV-derived, RVF-associated module contained 279 transcripts, of which 245 were protein-coding genes, 30 were potentially novel transcripts, and 4 were noncoding RNAs (1 long intergenic noncoding RNA, 1 pseudogene, 1 regulatory RNA, and 1 antisense RNA). These 279 transcripts displayed an average of 6.9 connections per transcript (Figure 1). GeneAnalytics revealed that the module was enriched in genes involved in striated muscle contraction, cytoskeletal signaling, fMLP ( $N$-formyl-Met-Leu-Phe) signaling, receptor tyrosine kinase EphB-EphrinB signaling, oxidative stress response, and protein metabolism (Supplemental Data Set 1). Other signaling pathways already known to be involved in HF - PKA signaling, PI3K/Akt signaling, Wnt signaling, and AMPK signaling — were also highlighted. Strikingly, the RVF-associated module appeared trilobed in structure. Two of the 3 lobes had strong, distinct physiological themes muscle filament sliding and striated muscle contraction (cardiac signaling lobe, Supplemental Data Set 2); and neutrophil degranulation and innate immune system signaling (innate immunity signaling lobe, Supplemental Data Set 3). The cardiac signaling lobe was also enriched in genes involved in cytoskeletal signaling, cell death (apoptosis and autophagy), and intracellular membrane transport. The innate immune system signaling lobe was additionally enriched in genes involved in cytokine signaling, cell chemotaxis, and PLC signaling. The third lobe of the RVF-associated module was moderately enriched in genes involved in Notch signaling, fMLP pathway, metabolism, calcium homeostasis, and ER stress (intracellular signaling lobe, Supplemental Data Set 4).

WIPI1, HSPB6, MAP4, SNAP47, and PRDX5 are potential determinants of RVF. To elucidate the mechanisms by which the RVF-associated module may regulate RVF, we focused on (a) those genes with high connectivity to other genes (hubs) and (b) those with high positive or negative correlations to hemodynamic indices of RVF (drivers or repressors). Of the 10 hubs, only WIPI1 encoding autophagy and mitophagy WD repeat domain phosphoinositide-interacting protein 1 was (a) differentially expressed in RV of BiV-HF 

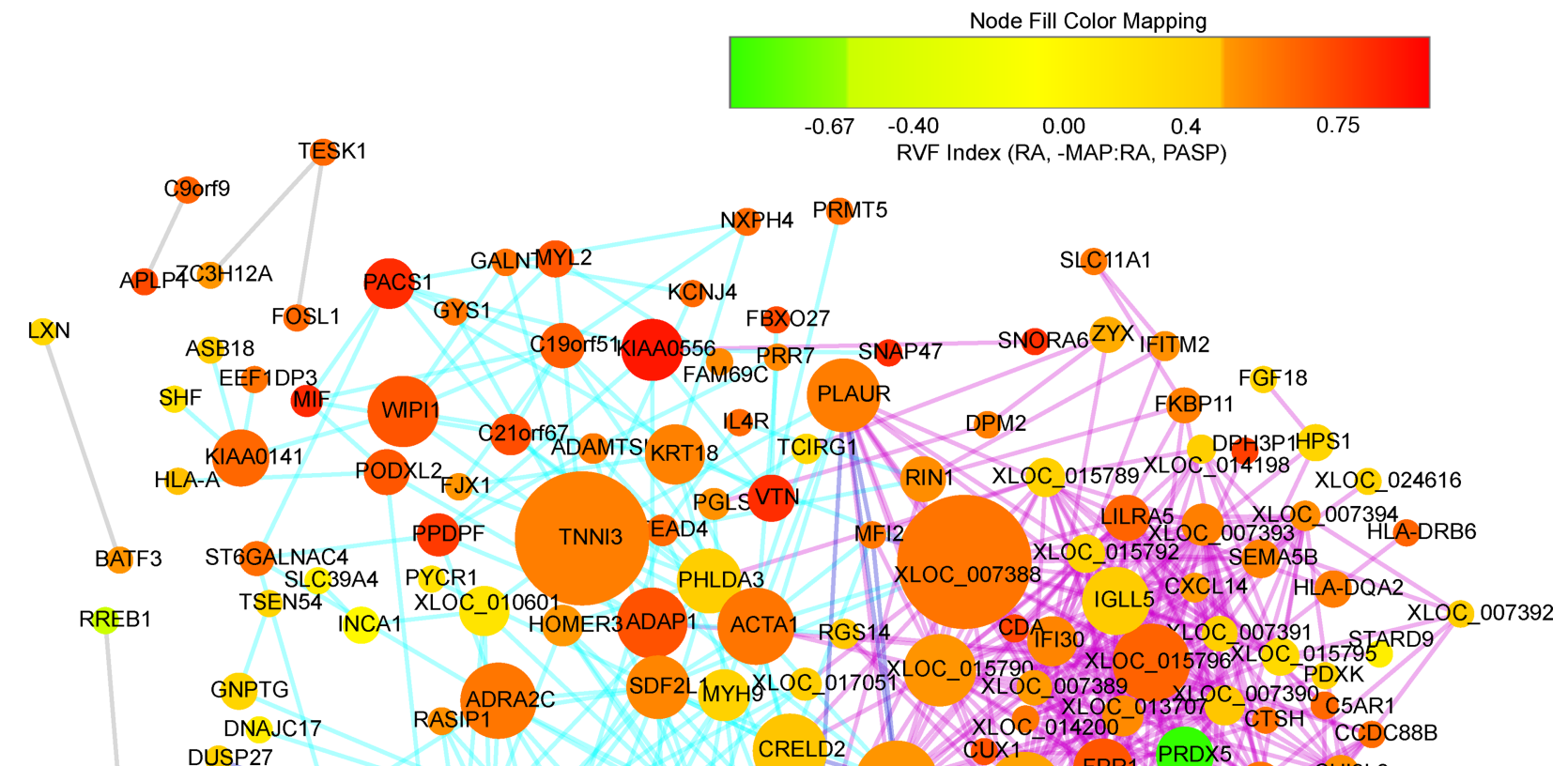

RBPMS

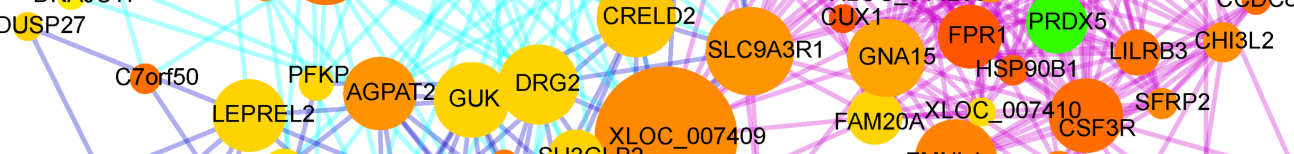

CSTB
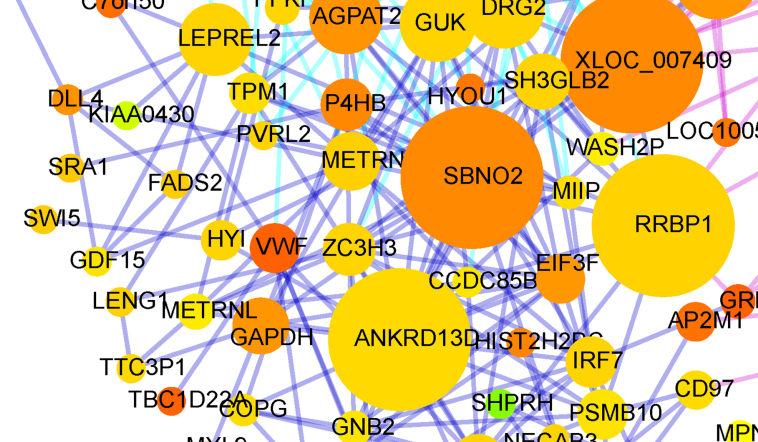

FAM20A

ALOC_007410 SFRP2 
Table 1. Pearson's correlation coefficients of RVF-associated drivers, repressor, and hub with hemodynamic indices

\begin{tabular}{|c|c|c|c|c|c|c|c|}
\hline \multirow{2}{*}{ Gene } & \multicolumn{7}{|c|}{ Correlation Coefficient (Pearson's P value) } \\
\cline { 2 - 8 } & RA & RA/PCWP & MAP/RA & PASP & SBP & CI & RVF Index \\
\hline HSPB6 & $0.810(<0.001)$ & $0.803(<0.001)$ & $-0.583(0.023)$ & $0.861(<0.001)$ & $-0.642(0.010)$ & $-0.688(0.005)$ & 0.75 \\
\hline SNAP47 & $0.735(0.002)$ & $0.727(0.002)$ & $-0.689(0.005)$ & $0.686(0.005)$ & $-0.761(0.001)$ & $-0.575(0.025)$ & 0.70 \\
\hline MAP4 & $0.703(0.003)$ & $0.592(0.020)$ & $-0.500(0.058)$ & $0.746(0.001)$ & $-0.697(0.004)$ & $-0.624(0.013)$ & 0.65 \\
\hline PRDX5 & $-0.679(0.009)$ & $-0.660(0.007)$ & $0.600(0.019)$ & $-0.630(0.012)$ & $0.602(0.018)$ & $0.745(0.001)$ & -0.64 \\
\hline WIP11 & $0.528(0.043)$ & $0.477(0.072)$ & $-0.455(0.088)$ & $0.664(0.007)$ & $-0.624(0.013)$ & $-0.442(0.099)$ & 0.55 \\
\hline
\end{tabular}

RA, right atrial pressure; RA/PCWP, ratio of right atrial pressure to pulmonary capillary wedge pressure; MAP/RA, ratio of mean arterial pressure to right atrial pressure; PASP, pulmonary artery systolic pressure; SBP, systolic blood pressure; $\mathrm{Cl}$, cardiac index; RVF, right ventricular failure; RVF index is calculated as the average of three coefficients - the correlation coefficients for RA and PASP and the negative value of the correlation coefficient for MAP/RA. Pearson's $P$ values are presented in parentheses.

hearts versus the RV of either LVF or NF hearts, and (b) differentially expressed in RV versus LV of BiV-HF hearts (Supplemental Table 3). Moreover, the expression of WIPI1 correlated with multiple RVF-associated hemodynamic indices (Table 1).

To identify genetic drivers and repressors of RVF, we examined the correlation of each of the 279 transcripts within the RVF-associated module to RA, RA/PCWP, MAP/RA, pulmonary artery systolic pressure (PASP), SBP, and CI (Supplemental Data Set 5). Increased HSPB6, SNAP47, and MAP4 expression and decreased PRDX5 expression were associated with increased RA, PASP, and RA/PCWP and with decreased MAP/RA, SBP, and CI — consistent with RVF (Table 1).

Wipil, Hspb6, and Map4 are upregulated only in the failing $R V$ and not in the merely dysfunctional $R V$. To validate their associations with RVF, we measured the ventricular expression of Wipi1, Hspb6, Snap47, Map4, and Prdx5 in a mouse model of pressure overload-induced RVF. Adult male C57BL/6J mice (age 10-12 weeks) were subjected to moderate pulmonary artery banding (PAB, $25 \mathrm{~g}$ ) or thoracotomy alone (sham) and assessed at 3-week intervals following surgery. By 3 weeks after PAB, RV systolic dysfunction and mild RV dilatation were echocardiographically evident (Figure 2, A-D). However, RVF, defined as markedly elevated RV end-diastolic pressure, diminished RV stroke volume, and pathological evidence of elevated central venous pressure in the form of hepatic congestion (increased liver weight/tibia length), did not manifest until 9 weeks after PAB (Figure 2, E and F). By 9 weeks after surgery, PAB mice (PAB9wk) also developed mild pulmonary edema (increased lung weight/tibia length) and peripheral edema (increased BW/tibia length). Failing PAB9wk mice also demonstrated marked induction of the fetal gene program, namely upregulation of atrial natriuretic factor ( $N p p a)$, brain natriuretic peptide ( $N p p b)$, and skeletal $\alpha$ actin (Acta1), and switching of cardiac myosin heavy chain isoforms to a predominance of $\beta \mathrm{MHC}$, encoded by $M y h c 7$ (Figure 2G).

As in the human BiV-HF hearts, Wipi1, Hspb6, Snap47, and Map4 mRNA expression was increased in the failing RV of PAB mice (PAB9wk-RV) compared with that of time-matched sham (Sham9wk-RV) (Figure 3A). Transcriptional analyses at 3- and 6- weeks after surgery confirmed that Wipi1, Hspb6, and Map4 inductions were indeed specific to RVF (PAB9wk) and not associated with simply RV pressure overload or RV dysfunction. Western blot analysis confirmed increased protein expression at PAB9wk of WIPI1, HSPB6, and MAP4 but not SNAP47 (Figure 3, B and C). In contrast to the findings in human BiV-HF RV, there was increased Prd $x 5$ transcript expression but no difference at the protein level between PAB9wk-RV and Sham9wk-RV.

Transcriptional upregulation of Wipil, Hspb6, and Map4 are specific to the failing RV and not evidenced in the failing $L V$. To confirm that these transcriptional changes were specific to the failing RV and not shared by the failing $\mathrm{LV}$, we also assessed the expression of these gene hub and drivers/repressors in a mouse model of pressure overload-induced LVF (Supplemental Figure 2). Adult male C57BL/6J mice (age 10-12 weeks) were subjected to severe transverse aortic constriction (TAC, $27 \mathrm{~g}$ ) or sham and assessed at 3-week intervals following surgery. By 3 weeks after surgery, TAC mice developed increased LV mass and depressed LV systolic function. By 6 weeks after surgery, TAC mice (TAC6wk) progressed to severe LV dysfunction, LV dilatation, and overt LVF, as manifested by severe pulmonary edema on terminal morphometric analysis; induction of the fetal gene program was also confirmed. Expression of Wipi1, Hspb6, Snap47, Map4, and Prdx5 in the failing LV of TAC6wk mice was similar to that in LV of Sham6wk mice. 

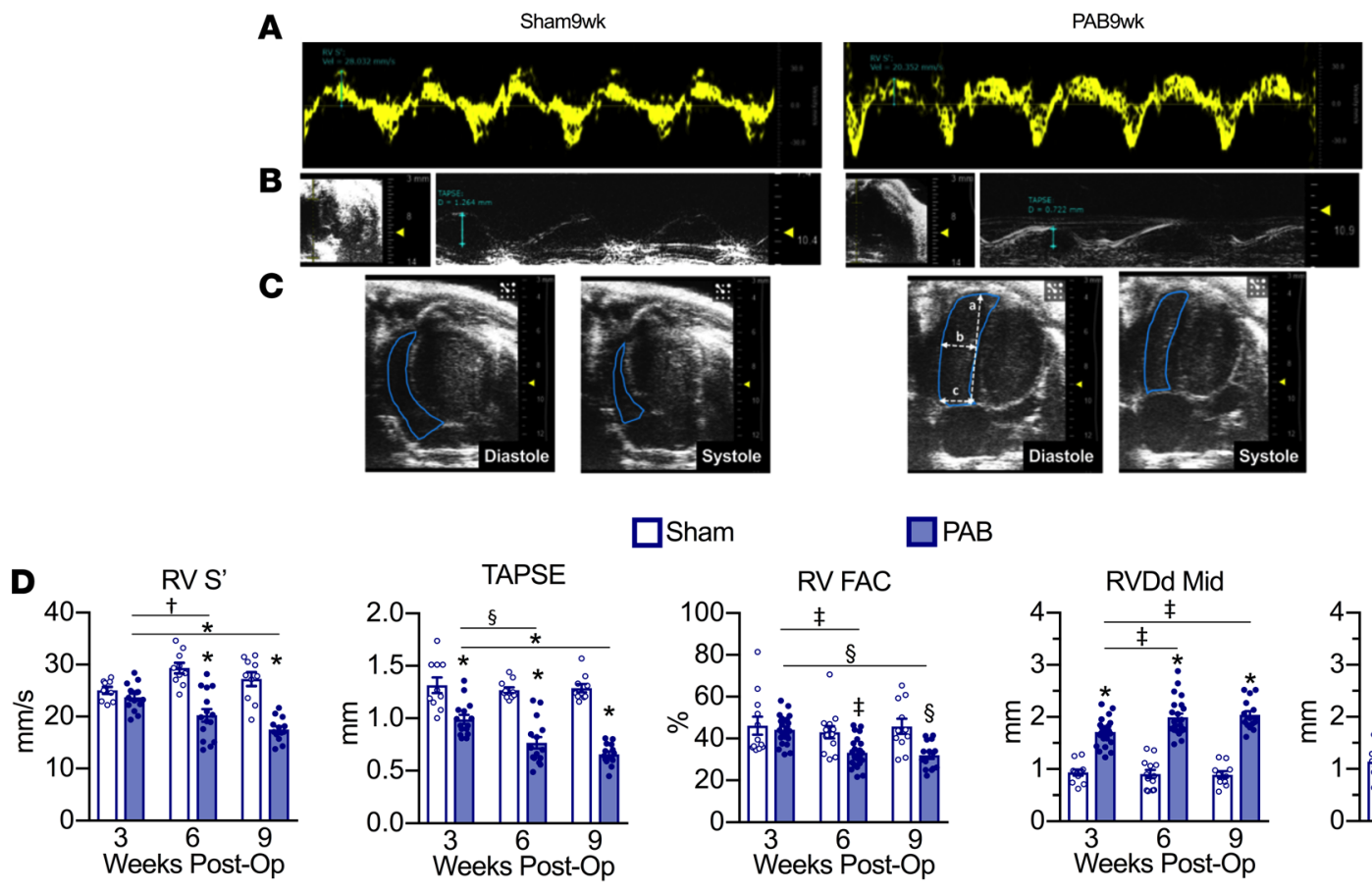

Sham

PAB
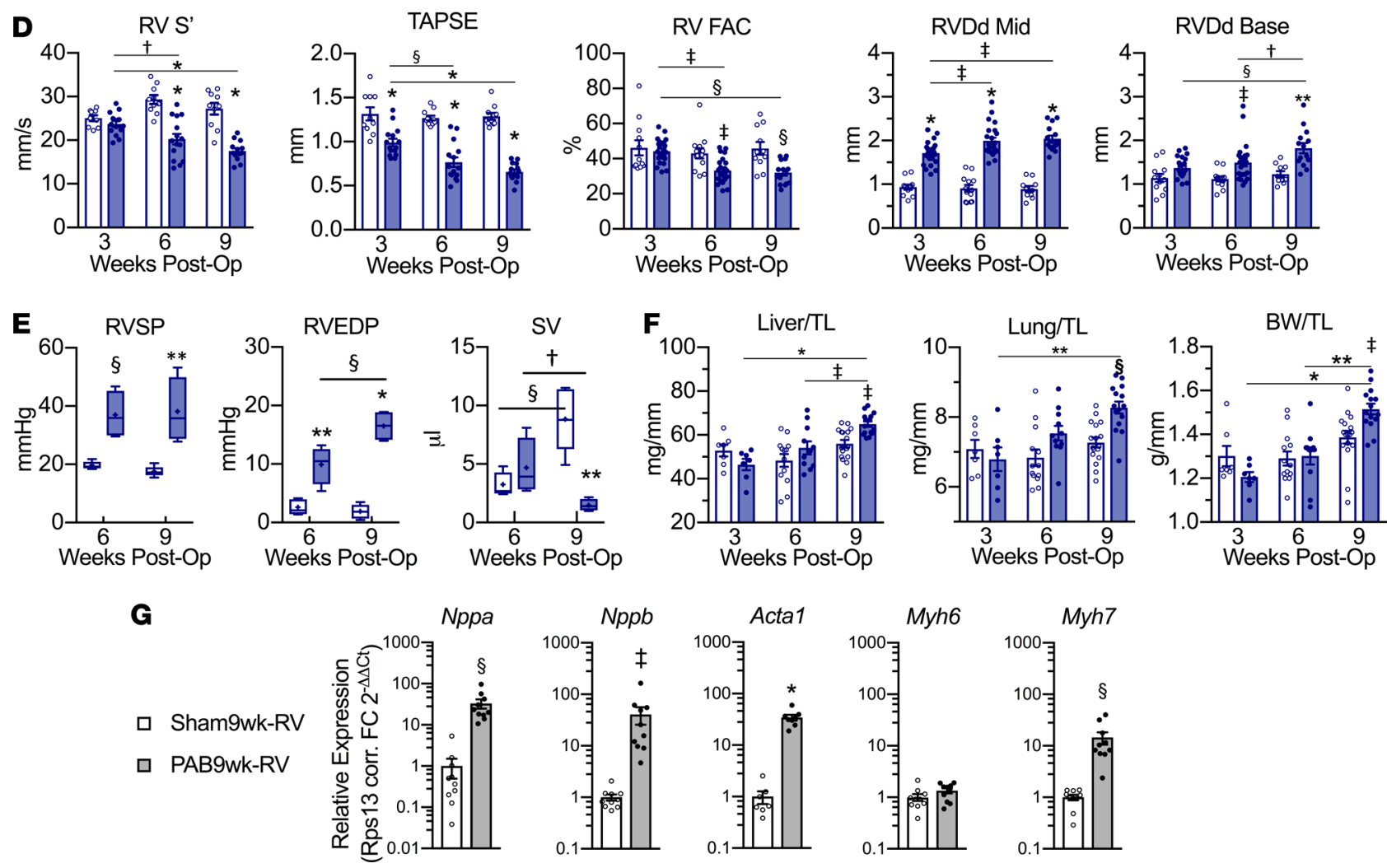

Figure 2. Pulmonary artery banding (PAB) induces progressive dilatation, functional decline, and eventual failure of the right ventricle (RV) over a 9-week course. [57BL/6] WT male mice were subjected to sham or PAB surgery and assessed at 3-week intervals, up to 9 weeks after surgery. Representative echocardiographic images of (A) tissue Doppler assessment of RV systolic function by lateral tricuspid annular systolic velocity $\mathrm{S}^{\prime}$, (B) M-mode assessment of TAPSE (tricuspid annulus planar systolic excursion), and (C) B-mode assessment of RV FAC (fractional area change) and RV diastolic dimensions (a, major axis dimension; b, mid-cavity dimension; c, basal dimension). (D) Summary of echocardiographic assessment of RV function and structure, $n=10-15$ per group.

(E) Terminal hemodynamic assessment of RV pressures (RVSP, RV peak systolic pressure; RVEDP, RV end-diastolic pressure) and stroke volume (SV) at 6 and 9 weeks after surgery for PAB and sham mice, $n=5$ per sham group, $n=4$ per PAB group. (F) Morphometric assessment of hepatic congestion (Liver/TL, liver weight/tibia length), pulmonary edema (Lung/TL, lung weight/tibia length), and peripheral edema (BW/TL), $n=7-17$ per group. (G) RT-qPCR analysis of fetal gene program in RV myocardium of PAB9wk versus Sham9wk, $n=7-10$ per group. ${ }^{*} P<0.0001,{ }^{\dagger} P<0.1,{ }^{\S} P<0.01,{ }^{\ddagger} P<0.05,{ }^{*} P<0.001$ versus respective sham unless otherwise indicated by comparison bar, by Tukey's multiple-comparison testing following 2-way ANOVA for panels D-F; by 2-tailed, unpaired Student's $t$ test for panel G. Scatter dot plots with bars show individual values and mean \pm SEM. Box-and-whisker plots show mean (+), median (midline), 25 th and 75 th percentiles (box), and minimum and maximum values.

In vitro silencing of Wipi1 partially protects against RVF-associated neurohormone-induced HF. WGCNA discovered modest to strong correlations between RVF-associated hub WIPI1 and each of our candidate RVF-associated drivers and repressors, suggesting potential functional or biological interactions between them $\left(\right.$ MAP4 $\mathrm{R}^{2}=0.802$, SNAP47 $\mathrm{R}^{2}=0.726$, HSPB6 $\mathrm{R}^{2}=0.672$, and PRDX5 $\left.\mathrm{R}^{2}=0.608\right)$. Our in vivo RVF mouse model validated that transcriptional changes in Wipil correlated with that of Hspb6 and Map4, thereby raising 
A

Sham-RV

口 PAB-RV
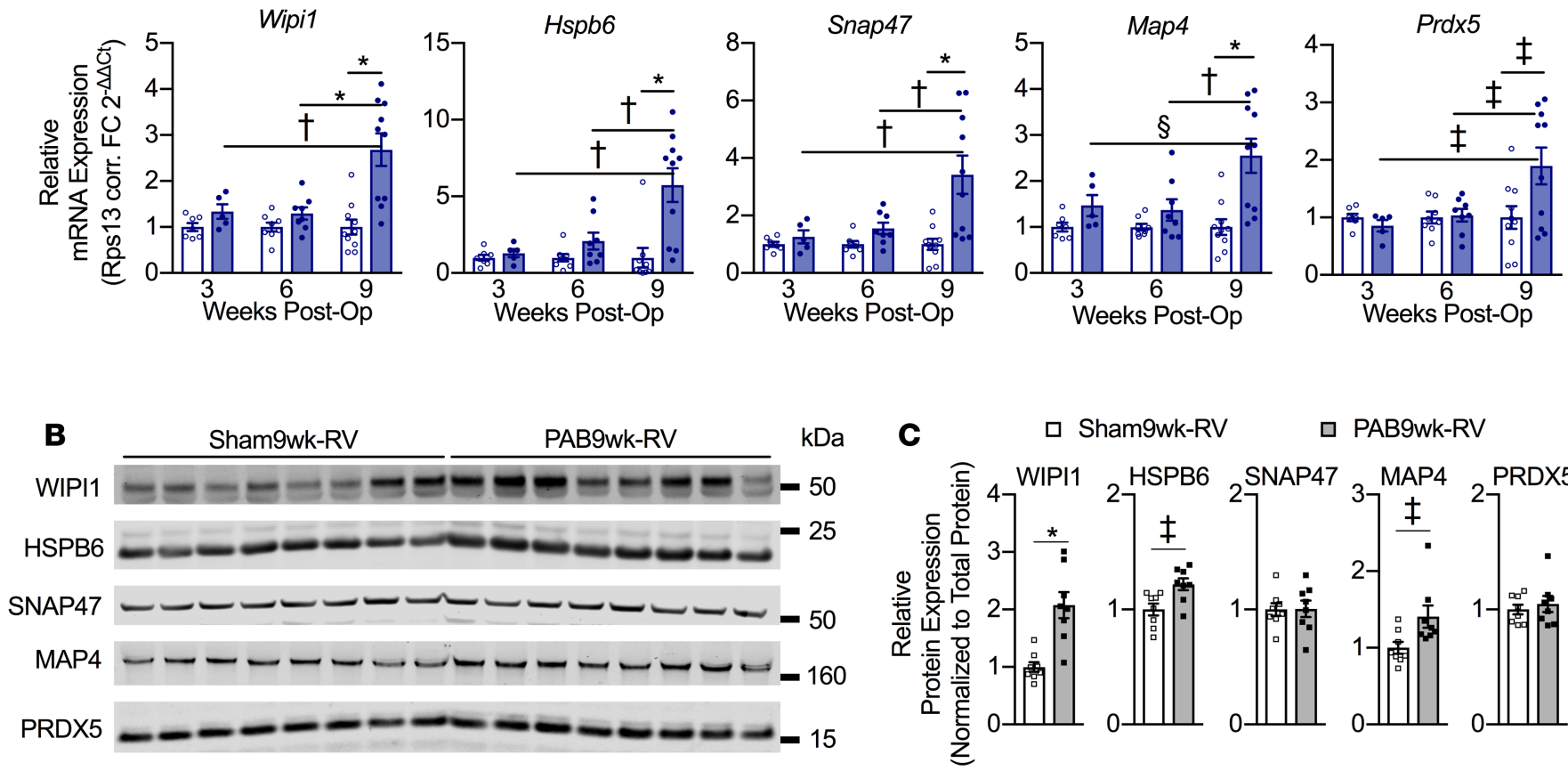

口 PAB9wk-RV

Figure 3. Transcript and protein expression of WGCNA-identified, RVF-associated gene hub, drivers, and repressor in pulmonary artery banding (PAB) mouse model. RNA and protein were extracted from the RV of C57BL/6) WT male mice subjected to sham or PAB. (A) RT-qPCR analysis of Wipi7, Hspb6, Snap47, Map4, and Prdx5 in RV of sham and PAB mice at 3, 6, and 9 weeks after surgery. (B) Representative Western blots, and (C) summary densitometry analysis of Westerns, normalized to total protein stain, relative to sham control. Total protein stain not shown. ${ }^{*} P<0.001,{ }^{\dagger} P<0.01,{ }^{\ddagger} P<0.05,{ }^{s} P=0.06$ by Tukey's multiple-comparison testing following 2-way ANOVA for panel A; by 2-tailed, unpaired Student's $t$ test for panel C. $n=5-10$ per group. Scatter dot plots with bars show individual values and mean \pm SEM.

the hypothesis that Wipil might potentially regulate one or both of these RVF-associated drivers. As a genetic hub of RVF, Wipil may be a potential target for RVF therapy. Thus, we sought to ascertain the cardioprotective potential of silencing Wipil in an in vitro model of HF and its effect on RVF-associated drivers. Specifically, we cultured isolated neonatal rat ventricular myocytes (NRVMs) with aldosterone to mimic the neurohormonal activation predominantly associated with RV dysfunction and failure (23-26). In control NRVMs transfected with nontargeting siRNA (si-Scramble), neurohormonal stimulation with aldosterone significantly increased the expression of Nppa, Nppb, Actal, and Myh7, consistent with induction of the fetal gene program in HF (Supplemental Figure 2). Specific siRNA (si-WipiI) significantly knocked down Wipil expression in NRVMs by approximately $70 \%$ at both the mRNA and protein levels (Figure 4). Notably, silencing Wipil prevented aldosterone-induced upregulation of $M y h 7$, suggesting that silencing Wipil may be partially protective against the neurohormone activation associated with RVF (Supplemental Figure 3).

Wipil regulates Map4 expression under conditions of aldosterone activation. To determine whether RVF hub Wipil might regulate Hspb6, Map4, Snap47, and/or Prdx5, we also assessed the mRNA and protein expression of these genes in si-Wipil-transfected versus si-Scramble-transfected NRVMs. The effect of silencing Wipi1 on Map4, Hspb6, Snap47, and Prdx5 varied. Silencing Wipi1 in NRVMs decreased Map4 transcript expression by $25 \%-30 \%$ relative to its expression in si-Scramble control NRVMs, under both basal and aldosterone-stimulated conditions (Figure 4, A and B). Aldosterone did not affect Map4 transcript levels in either si-Wipil or si-Scramble NRVMs. However, aldosterone did induce MAP4 protein expression in si-Scramble control NRVMs. This aldosterone-induced upregulation of MAP4 protein expression was not observed in si-Wipi1 NRVMs (Figure 4C). In contrast, silencing Wipil had no impact on Hspb6 transcript expression. Aldosterone stimulated Hspb6 transcript expression in both si-Wipil and si-Scramble NRVMs. HSPB6 protein expression was consistent with transcript data. Neither silencing Wipil nor stimulating with aldosterone affected Snap 47 or Prdx 5 transcript or protein expression. Taken together, our findings validated the WGCNA-calculated correlation between Wipil and Map4 transcripts.

Wipil upregulation correlates with increased noncanonical autophagy in the failing $R V$. Because WIPI1 has been implicated in early autophagosome formation $(27,28)$, we investigated whether autophagy might 
$\mathbf{A}$

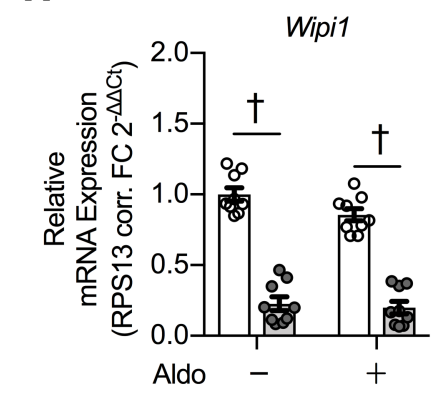

- si-Scr

- si-Wipi1
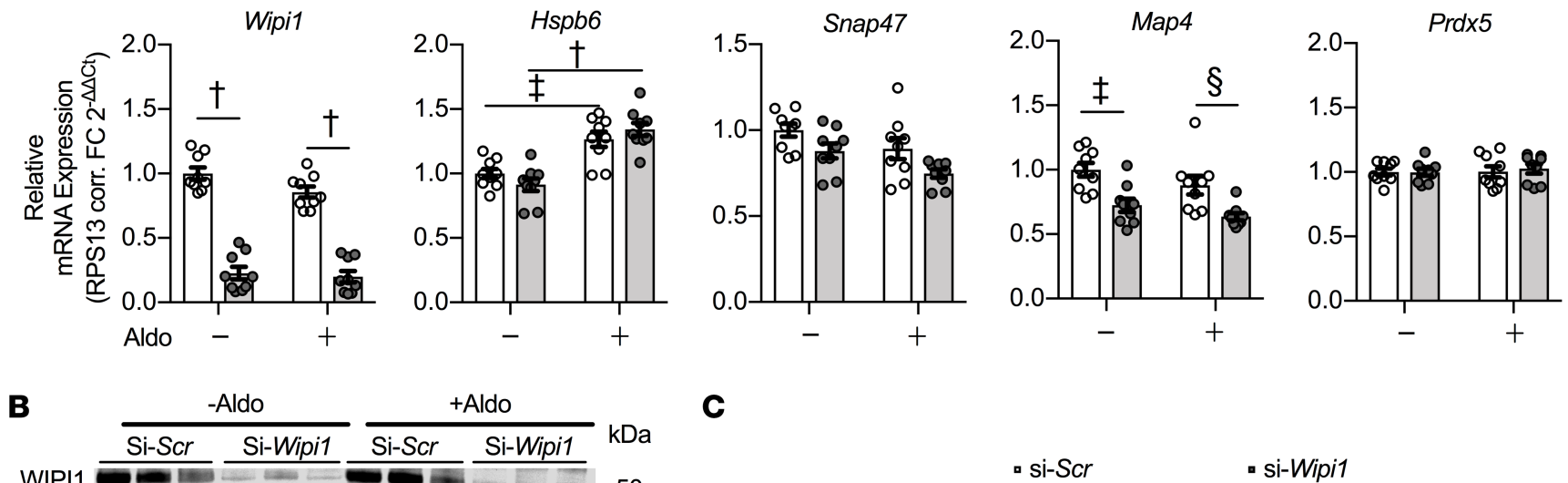

\section{c}
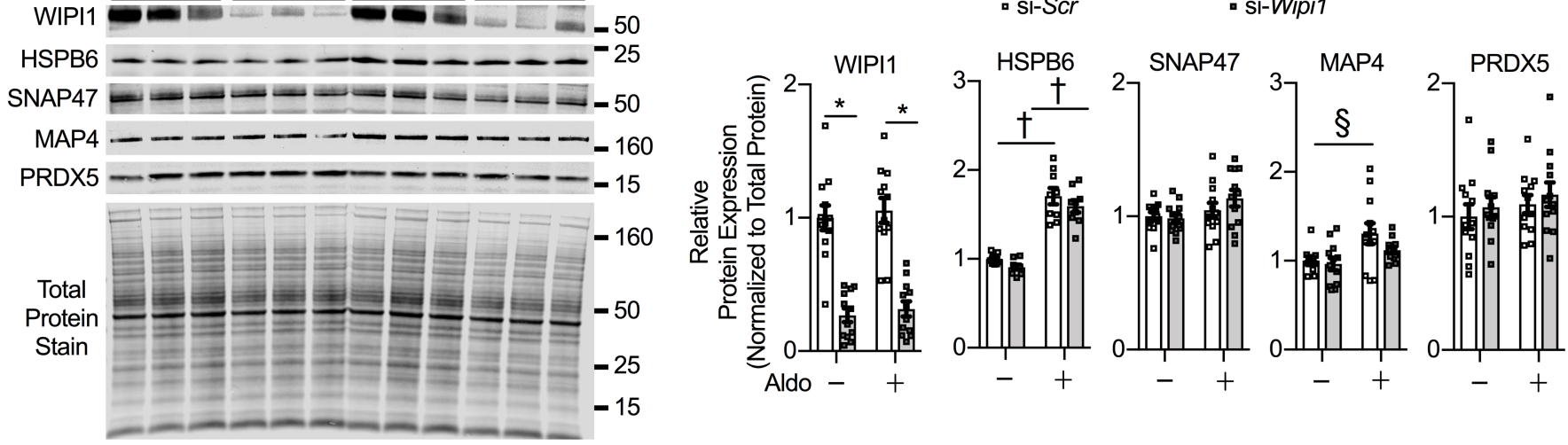

Figure 4. Effect of silencing Wipi1 on hub, drivers, and repressor of WGCNA-identified RVF-associated module. Neonatal rat ventricular myocytes (NRVMs) were transfected with scramble or Wipi1-specific siRNAs and then stimulated with aldosterone (Aldo, $1 \mu \mathrm{M})$ for 48 hours. (A) Effect of Aldo stimulation and Wipi1 silencing on transcript levels of WCCNA-identified RVF-associated genetic hub, drivers, and repressor ( $n=9$ per group from 3 independent experiments). (B) Representative Western blot. (C) Summary of Western analysis ( $n=9-12$ per group from $3-4$ independent experiments). ${ }^{*} P<0.001,{ }^{\dagger} P<0.0001,{ }^{\ddagger} P<0.01$, $\S P<0.05$ by Tukey's multiple-comparison testing following 2-way ANOVA. Scatter dot plots with bars show individual values and mean \pm SEM.

be differentially dysregulated in the failing RV versus the failing LV. An autophagy-focused heatmap of our human ventricular transcriptomic data suggested differential dysregulation of autophagy pathways in the failing RV versus the failing LV (Supplemental Figure 4). To substantiate this hypothesis, we sought to characterize autophagy in our mouse models of RV versus LVF. We analyzed the expression of autophagy proteins beclin-1 (BECN1) and microtubule-associated protein light-chain 3 (LC3), the ratio of the phosphatidylethanolamine conjugate to the cytosolic isoform of LC3 (LC3-II/I) as an index of LC3-lipidation and autophagic flux (29), and phosphorylation of serine-16 of heat shock protein B6 (HSPB6) as a marker of canonical autophagy (30) (Figure 5A). BECN1, HSPB6, WIPI1, and LC3I were all upregulated in PAB9wk-RV compared with Sham9wk-RV (Figure 5B). However, LC3II expression and the LC3-II/I ratio remained similar in PAB9wk-RV and Sham9wk-RV. Coupled with the finding that Ser16-phosphorylated HSPB6/total HSPB6 ratio remained low and unchanged in PAB9wk-RV relative to Sham9wk-RV (Figure $5 \mathrm{C}$ ), these findings suggest that in the failing RV, noncanonical autophagy is upregulated while canonical autophagy is not. In contrast, in the failing TAC6wk-LV, both HSPB6 and its Ser16 phosphorylation were increased relative to levels in Sham6wk-LV (Supplemental Figure 5), suggesting an upregulation of canonical autophagy in the failing LV. Hence, noncanonical autophagy may play a more significant role in the pathophysiology of RVF, while p-Ser16-HSPB6-mediated, BECN1-dependent, canonical autophagy is more predominant in LVF.

Silencing Wipil prevents aldosterone-induced noncanonical autophagy in NRVMs. Given the central role for WIPI1 across multiple autophagy pathways $(31,32)$ and our finding of increased noncanonical autophagy in the RVF mouse model, we hypothesized that silencing Wipil might restore a physiological balance of autophagy pathways by blunting the excessive noncanonical autophagy associated with RVF. We assessed the effect of silencing Wipil on BECN1 expression, LC3 lipidation, and phosphorylation of HSPB6 Ser16 in our in vitro model of RVF-associated neurohormone activation. Isolated NRVMs were transfected with either si-Scramble 
A

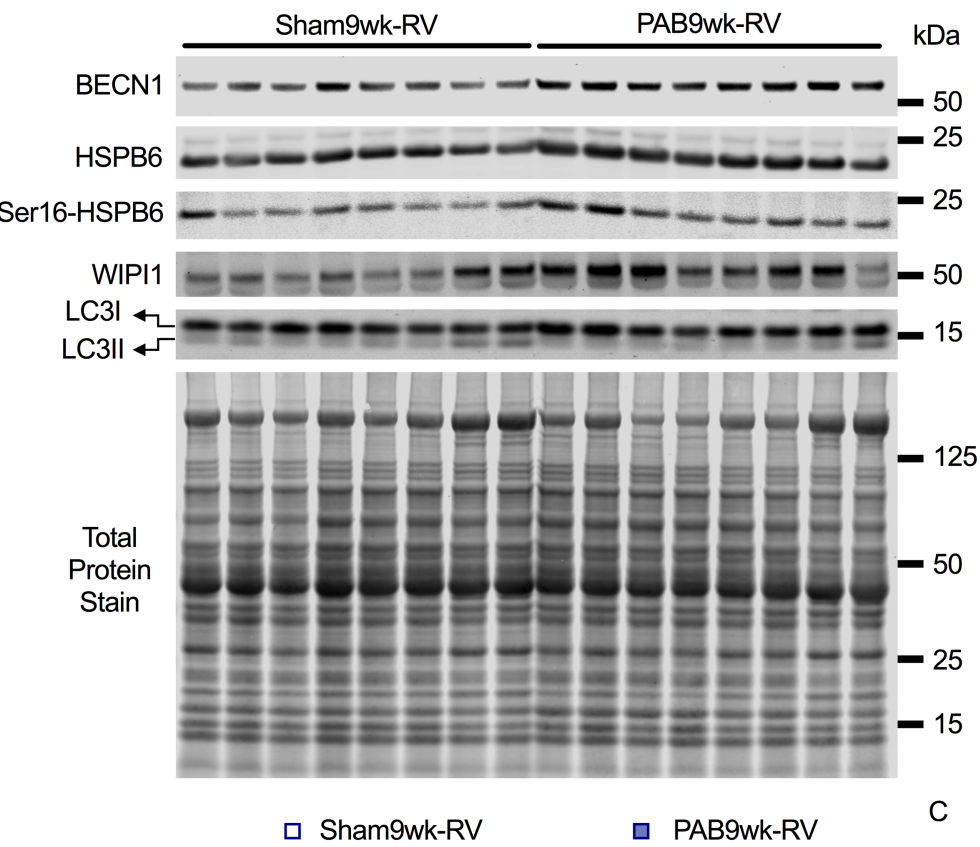

B

․ Sham9wk-RV

口 PAB9wk-RV

C
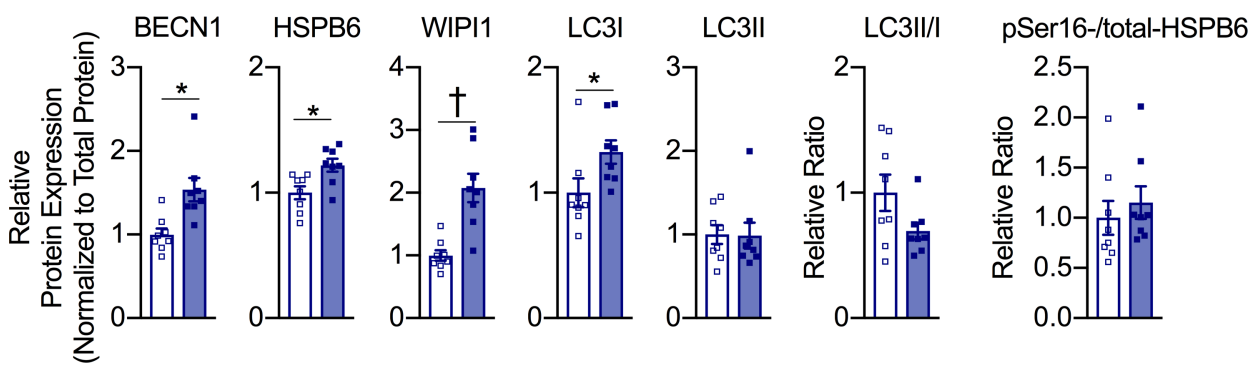

Figure 5. Noncanonical autophagy is upregulated in the failing right ventricle (RV) of pulmonary artery banding (PAB) mouse model. Protein lysates were prepared from RV of 9-week sham- or PAB-operated C57BL/6J WT male mice. (A) Western blots of autophagy proteins and total protein stain in nonfailing Sham9wk-RV and failing PAB9wk-RV. The HSPB6 and WIPI1 blots shown are reused from Figure 3B. (B) Summary of Western analyses reveals upregulation of BECN1, HSPB6, WIPI1, and nonlipidated LC3 (LC3I) without an increase in either LC3 lipidation (LC3II and LC3II/I ratio) or (C) phosphorylation of HSPB6 Ser16 in failing PAB9wk-RV versus nonfailing Sham9wk-RV. This suggests a shift towards noncanonical autophagy pathways in the failing RV. ${ }^{*} P<0.05,{ }^{\dagger} P<0.001$ by 2 -tailed Student's $t$ test; $n=8$ per group. Scatter dot plots with bars show individual values and mean $\pm \mathrm{SEM}$.

or si-Wipil and subsequently cultured for 48 hours, under serum-starved conditions, with or without aldosterone. In si-Scramble control NRVMs, aldosterone did not affect BECN1 expression or LC3 lipidation but did decrease Ser16-HSPB6 phosphorylation in si-Scramble NRVMs (Figure 6, A and B). These results suggest that aldosterone inhibits p-Ser16-HSPB6/BECN1-dependent (canonical) autophagy while increasing noncanonical autophagy in cardiac myocytes for a net constant overall autophagic flux. Under basal conditions, silencing Wipil had no affect on BECN1 expression, LC3 lipidation, or Ser16-HSPB6 phosphorylation. However, under aldosterone stimulation, silencing Wipil decreased LC3 lipidation (LC3II/I ratio) without affecting Ser16HSPB6 phosphorylation relative to the respective si-Scramble control. Hence, silencing Wipil could selectively limit noncanonical autophagy under conditions of chronic aldosterone activation.

To further elucidate the role of WIPI1 in canonical versus noncanonical autophagy, we used 2 distinct autophagy inhibitors to differentiate the effects of Wipil silencing on these autophagy pathways. V-ATPase inhibitor bafilomycin A (BafA) blocks LC3II lysosomal degradation during canonical autophagy. Chloroquine (CQ) inhibits the fusion between the autophagosome and lysosome, thereby rendering it capable of revealing total autophagic flux, inclusive of both canonical and noncanonical autophagy $(33,34)$. Thus, the difference between the effects of CQ versus BafA on LC3-II/I ratios is attributed to noncanonical autophagy. Here, we transfected NRVMs with si-Wipil versus si-Scramble, treated them with either BafA or CQ, and then measured LC3-II/I ratios (Figure 6C). BafA increased LC3-II/I equally in si-Wipil- and si-Scramble-transfected 
A

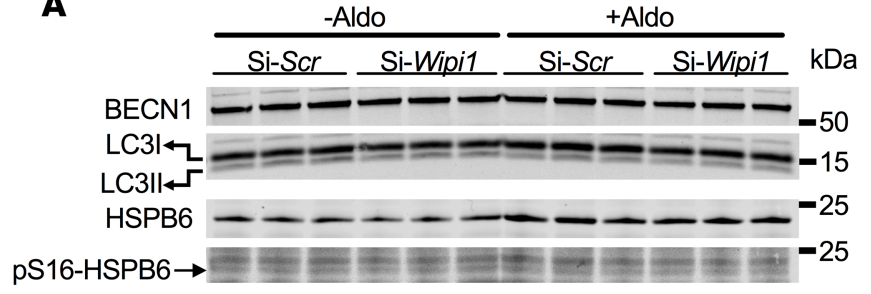

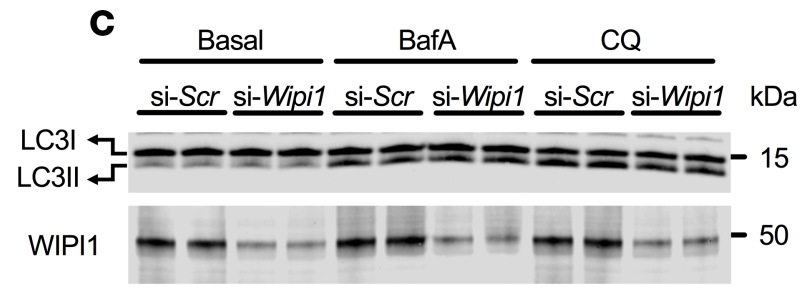

pS16-/total-HSPB6

D

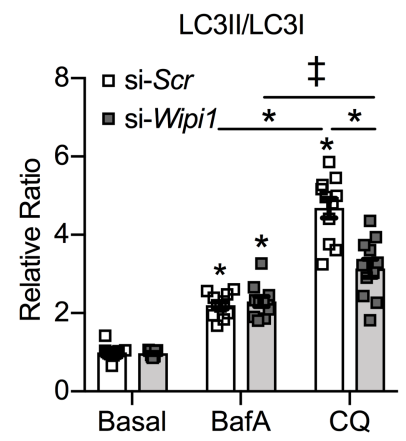

Figure 6. Silencing Wipi1 blunts aldosterone induction of noncanonical autophagy. Neonatal rat ventricular myocytes (NRVMs) were transfected with scramble or Wipi1-specific siRNAs and then stimulated with aldosterone (Aldo, $1 \mu \mathrm{M}, 48$ hours), bafilomycin A (BafA, $100 \mathrm{nM}, 1$ hour), or chloroquine (CQ, $100 \mu \mathrm{M}, 1$ hour). (A) Representative Western blots of autophagy proteins ( $n=9-18$ per group from 3-6 independent experiments). (B) Summary Western analysis of LC3 lipidation (LC3II/LC3I) and canonical autophagy (pS16-/total HSPB6). (C) Representative Western blots of LC3 and WIPI1 in si-Scramble- versus si-Wipi-transfected NRVMs treated with BafA or CQ to differentiate, respectively, canonical versus noncanonical autophagy. BafA blocks LCII Iysosomal degradation during canonical autophagy, whereas CQ inhibits the fusion between the autophagosome and lysosome. Hence, CQ reveals total autophagic flux and the difference between the effects of CQ and BafA on LC3II/I ratios is attributable to noncanonical autophagy (32, 33). (D) Quantification of LC3II/I ratio shows that silencing Wipi1 selectively inhibits noncanonical autophagy (CQ) without affecting canonical autophagy (BafA) ( $n=11$ per group from 4 independent experiments for $L C 3 I I / I) .{ }^{*} P<0.0001,{ }^{\ddagger} P<0.01,{ }^{\dagger} P<0.05$ versus basal, unless otherwise indicated by comparison bar, by Tukey's multiple-comparison test following 2-way ANOVA. Scatter dot plots with bars show individual values and mean \pm SEM.

NRVMs, suggesting that canonical autophagy remains intact even when Wipil is silenced. However, silencing Wipil blunted the CQ-induced increase in the LC3-II/I ratio otherwise seen in si-Scramble-transfected NRVMs (Figure 6D), suggesting that Wipil plays a substantial role in noncanonical autophagy.

Silencing Wipil blunts aldosterone induction of mitochondrial superoxide and mitochondrial protein oxidation. Recent studies have revealed an emerging relationship between mitochondrial oxidative stress and autophagy $(35,36)$. Thus, we investigated the effect of silencing Wipil on mitochondrial superoxide levels in NRVMs subjected to aldosterone treatment. Aldosterone increased mitochondrial superoxide levels in si-Scramble control NRVMs, as observed with the MitoSOX Red superoxide indicator (Figure 7, A and B). Silencing Wipil blunted aldosterone-induced mitochondrial superoxide but not that induced by hydrogen peroxide $\left(\mathrm{H}_{2} \mathrm{O}_{2}\right)$. Neither aldosterone stimulation nor silencing Wipil negatively impacted cell viability (Figure 7C). Moreover, subsequent assessment of the redox state of mitochondrial proteins cyclophilin D (CYPD) and thioredoxin 2 (TRX2) confirmed that silencing Wipil prevents downstream oxidation of these mitochondrial proteins (Figure 8). Silencing Wipil blunted aldosterone-induced oxidation of CYPD and TRX2 but had no effect on $\mathrm{H}_{2} \mathrm{O}_{2}$-induced protein oxidation (Figure 8, B-D). Altogether, these results indicate that silencing Wipi1 not only protects cardiac myocytes from excessive noncanonical autophagy but also mitigates mitochondrial oxidative stress by blunting mitochondrial superoxide levels and limiting mitochondrial protein oxidation.

\section{Discussion}

RVF portends accelerated clinical decline and early death in patients with cardiac or pulmonary disease, and yet no therapies exist that directly target the RV. The goal of this study was to leverage human transcriptomic data to identify myocardial determinants of RVF and experimentally validate candidate genes in in vivo and in vitro models of RVF. Using an unbiased and robust, large-scale, module-based statistical approach, we identified WIPI1 as a genetic hub of RVF, experimentally validated it in mouse models, and tested hypotheses regarding its pathophysiological role in isolated cardiac myocytes under conditions mimicking the neurohormonal activation of RVF. We provide insights into the role of Wipil in noncanonical autophagy and 
A
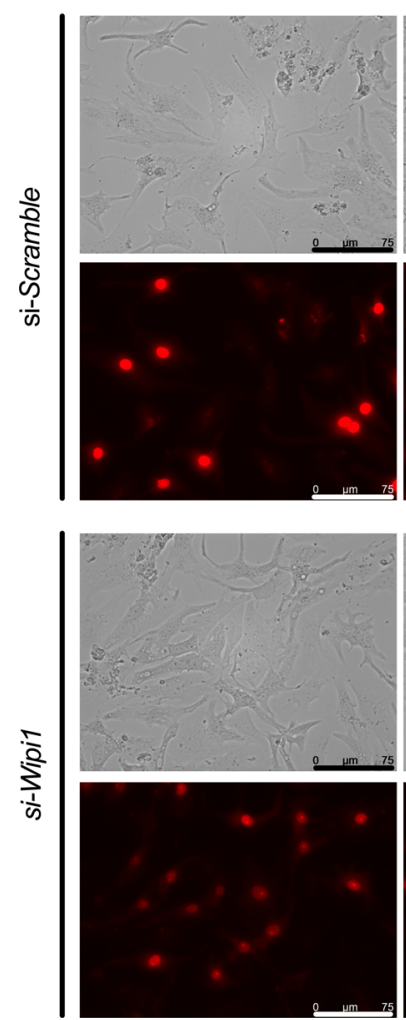

Aldosterone
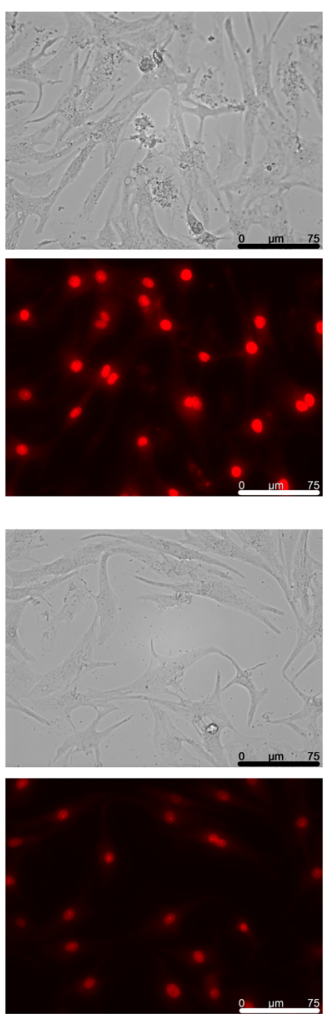
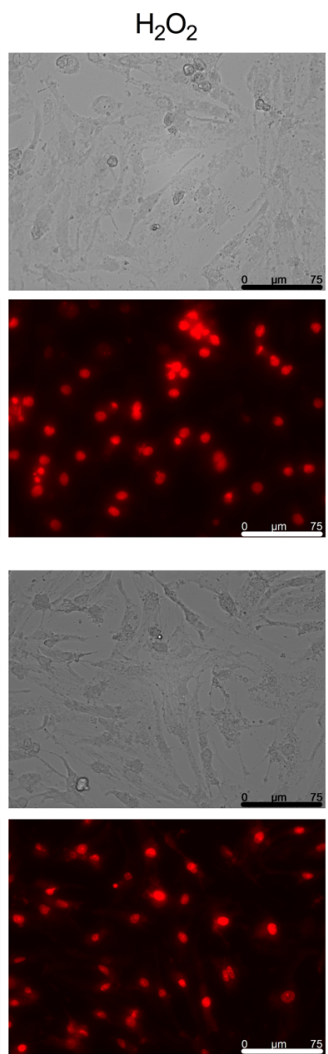

B

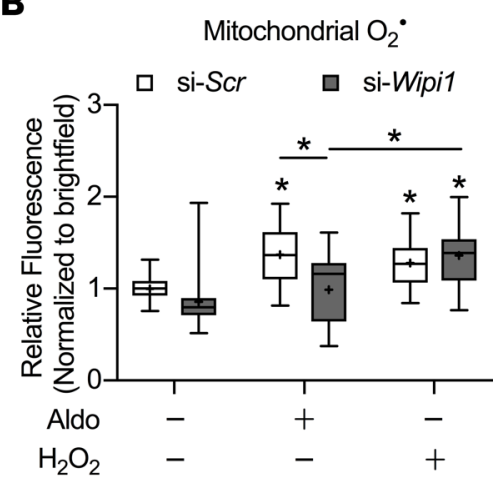

C

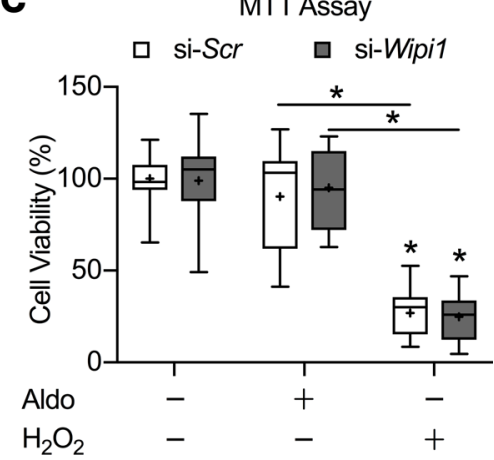

Figure 7. Silencing Wipi1 decreases mitochondrial superoxide $\left(\mathrm{O}_{2}\right)$ levels in in vitro neurohormonal model of right ventricular failure (RVF). Neonatal rat ventricular myocytes (NRVMs) were transfected with scramble or Wipi7-specific siRNAs and then stimulated with aldosterone (Aldo, $1 \mu \mathrm{M}, 48$ hours) or hydrogen peroxide ( $\mathrm{H}_{2} \mathrm{O}_{2}, 50 \mu \mathrm{M}, 2$ hours). (A) Brightfield and MitoSOX Red imaging of NRVMs transfected with si-Scramble versus si-Wipi1, with and without Aldo stimulation. $\mathrm{H}_{2} \mathrm{O}_{2}$ was used as a positive control. Scale bars: $75 \mu \mathrm{m}$. (B) Summary analysis of mitochondrial $\mathrm{O}_{2}$ ' levels $(n=41-44$ per group from 6 independent experiments). (C) Cell viability as assessed by MTT assay ( $n=24$ per group from 3 independent experiments). ${ }^{*} P<0.0001$ versus respective unstimulated baseline unless indicated otherwise by comparison bar, by Tukey's multiple-comparison test following 2-way ANOVA. Box-and-whisker plots show mean (+), median (midline), 25th and 75th percentiles (box), minimum and maximum values (whiskers).

in mitochondrial oxidative stress signaling. Our findings also offer proof of principle that silencing cardiac myocyte Wipil signaling holds therapeutic potential in RVF, by preventing excessive noncanonical autophagy and blunting mitochondrial superoxide levels and mitochondrial protein oxidation (Figure 9).

WIPI1 was first discovered for its role in nascent autophagosome formation and subsequently implicated in both canonical and noncanonical autophagy pathways (37). Although cardiac myocyte autophagy has been associated with human $\operatorname{HF}(38,39)$, the precise roles of canonical versus noncanonical autophagy in cardiovascular disease is unknown and unexplored. Transgenic mice with gain or loss of function of autophagy-related genes (40-43) and animal models of induced cardiac dysfunction (30, 35, 40, 41, 43-46) have demonstrated both cytoprotective and pathologic roles of autophagy in the heart. Moreover, both increased and decreased autophagic flux have been associated with LVF. Such ambiguity might be explained by differential dysregulation of canonical versus noncanonical autophagy (31). Whereas canonical autophagosome formation involves distinct hierarchical steps that require specific autophagy-related (ATG) proteins at each stage, noncanonical autophagosome formation does not require the involvement of all ATG proteins. Noncanonical autophagosome elongation may also occur from multiple membrane sources or from preexisting, nonphagophore endomembrane. Notably, noncanonical autophagy pathways include those that are independent of either BECN1 or LC3 lipidation. Prior cardiac autophagy studies often assessed autophagic flux via LC3 lipidation, a step universal to canonical and some noncanonical autophagy pathways as well as mitophagy, but did so without distinguishing these pathways. Both canonical and noncanonical autophagy are likely important for cellular homeostasis, and dysregulation of either or both pathways may underlie specific pathophysiological responses.

By incorporating analyses of a recently identified upstream regulator of canonical autophagy (i.e., p-Ser16 HSPB6) with that of LC3 lipidation, we provide evidence suggesting that increased noncanonical autophagy distinguishes the failing RV from the failing LV. Furthermore, we demonstrated in NRVMs that 
A
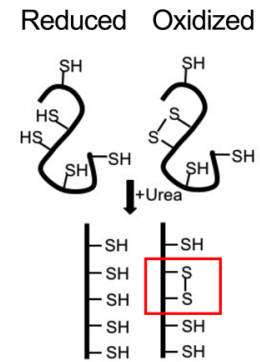

I $+\mid A M$

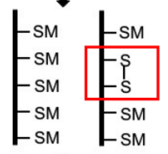

I+DTT

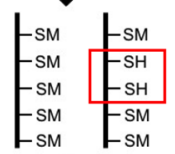

I + IAA

\begin{tabular}{l|l}
\multicolumn{1}{c}{${ }_{-S M}$} & $-S M$ \\
\cline { 2 - 2 }$-S M$ & $-S A$ \\
$-S M$ & $-S A$ \\
\hline$-S M$ & $-S M$ \\
\hline$-S M$ & $-S M$
\end{tabular}

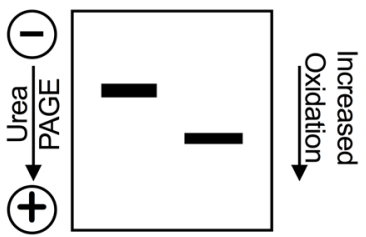

B
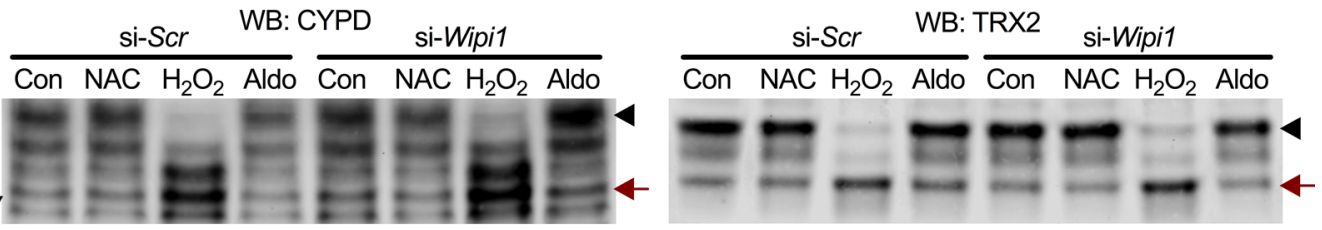

C
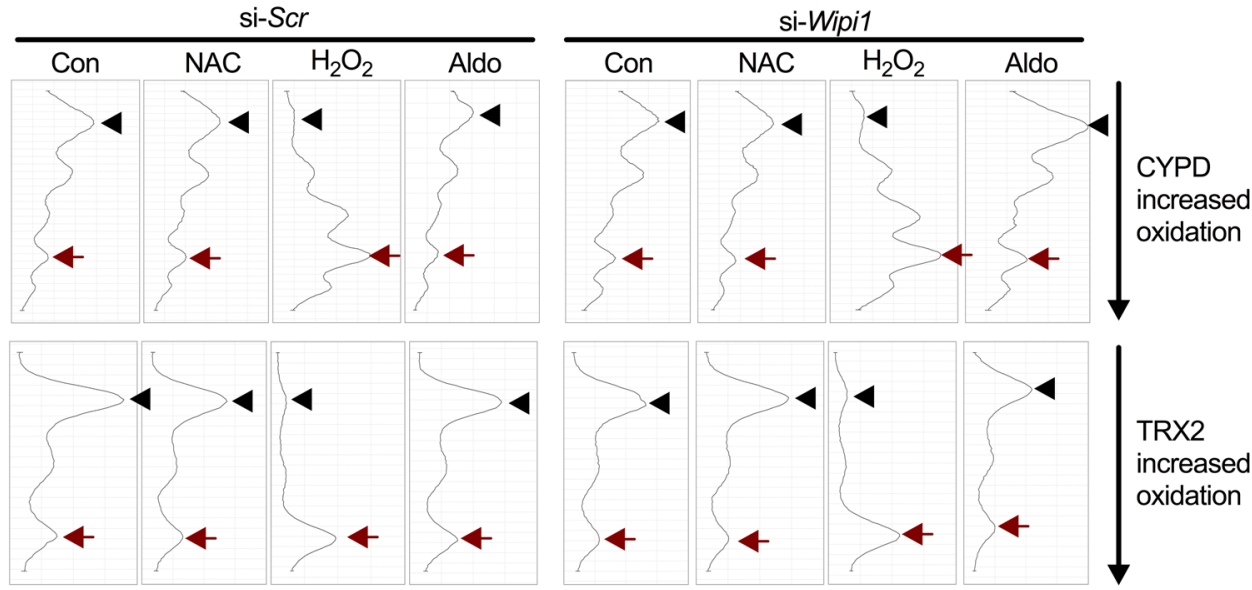

a si-Ser

si-Wipi1

TRX2

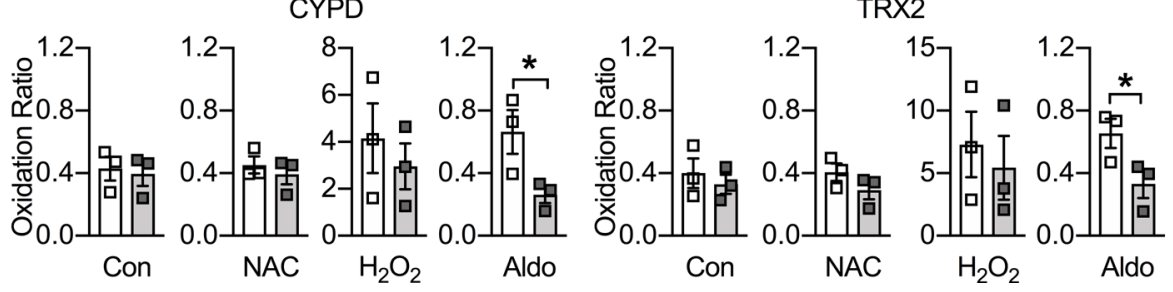

Figure 8. Silencing Wipi1 mitigates aldosterone-induced oxidation of mitochondrial proteins CYPD and TRX2. Neonatal rat ventricular myocytes (NRVMs) were treated with oxidizing or reducing agents and then subjected to urea lysis, iodoacetamide-iodoacetic acid (IAM-IAA) alkylation, and Western analysis. (A) Schematic representation of the IAM-IAA alkylation method for identifying oxidized and reduced proteins in native nonreducing urea PACE. (B) Representative redox Western blots for CYPD and TRX2 of NRVMs treated with (i) control, (ii) reducing agent $\mathrm{N}$-acetyl cysteine (NAC), (iii) oxidizing agent hydrogen peroxide ( $\mathrm{H}_{2} \mathrm{O}_{2}$ ), or (iv) aldosterone (Aldo). Black arrowhead, reduced protein band. Red arrow, oxidized protein band. (C) Histogram of Western densitometric analysis of CYPD and TRX2 oxidation. (D) Quantification of CYPD and TRX2 oxidation under different redox conditions as shown by the ratio of oxidized to reduced protein band signals ( $n=3$ per group). ${ }^{*} P<0.05$ by 1 -tailed Student's $t$ test. Scatter dot plots with bars show individual values and mean \pm SEM.

silencing Wipil can limit aldosterone-induced noncanonical autophagy while still permitting canonical autophagy. Our finding is significant as it provides evidence that these autophagy pathways can be differentially intervened upon under pathological conditions. Further studies are needed to elucidate the differential role of WIPI1 in noncanonical versus canonical autophagy.

Strikingly, our studies also suggest novel functions for WIPI1. We provide evidence that WIPI1 regulates mitochondrial oxidative stress signaling. Silencing Wipi1 in NRVMs decreased aldosterone-stimulated mitochondrial superoxide levels and limited oxidation of mitochondrial proteins CYPD and TRX2. The significance of this discovery is 2-fold. First, the RV is more vulnerable than the LV to oxidative stress due to interventricular differences in ROS regulation (47). Thus, attenuating mitochondrial oxidative stress, either through decreasing mitochondrial ROS production or improving antioxidant defense, is a promising therapeutic approach for RVF. Secondly, autophagy is known to regulate redox homeostasis; intracellular ROS triggers autophagy and mitophagy, which in turn modulate ROS levels. Dysregulated autophagy may potentiate detrimental ROS signaling. WIPI1 appears to lie at the nexus of autophagy and mitochondrial ROS signaling. How WIPI1 regulates mitochondrial superoxide surpasses the scope of our current study but warrants further investigation.

Another function of WIPI1 proposed by our studies relates to its observed correlation with MAP4 (microtubule-associated protein 4). WGCNA of human ventricular tissue revealed a strong correlation between WIPI1 and MAP4, which we then corroborated in our mouse model of RVF. We subsequently found 


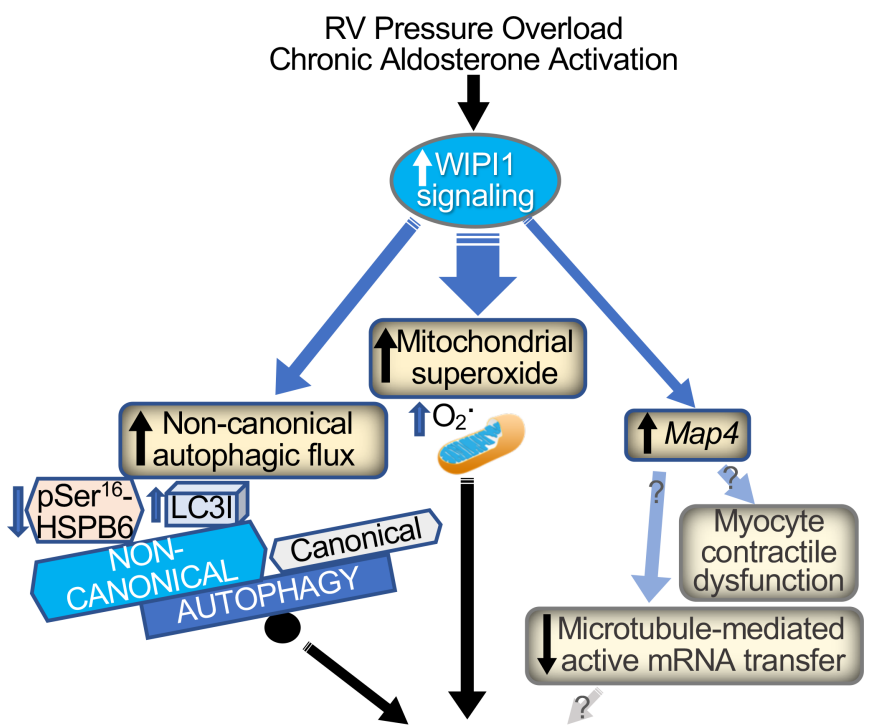

Right Ventricular Failure

Figure 9. Proposed schematic of Wipi1 signaling mechanisms underlying right ventricular failure (RVF). RV pressure overload and chronic aldosterone activation upregulate WIPI1 signaling in the failing RV. Enhanced WIPI1 signaling increases mitochondrial superoxide levels and noncanonical autophagic flux. WIPI1 upregulation also correlates with increased Map4 expression, thereby potentially triggering MAP4-mediated myocyte contractile dysfunction or inhibition of microtubule-mediated active mRNA transfer. In vitro studies in neonatal rat ventricular myocytes demonstrated the feasibility of mitigating aldosterone-induced mitochondrial superoxide levels, blunting noncanonical autophagy, and decreasing Map4 expression by silencing Wipi1. Further studies are warranted to elucidate mechanistic details of WIPI1 signaling and to confirm the therapeutic potential of targeting WIPI1 in RVF.

that silencing Wipil in NRVMs reduced Map4 transcript levels, at baseline and with aldosterone stimulation. MAP4 protein expression data in the NRVMs were not as definitive; MAP4 protein levels did not mirror all the changes observed at the transcript level, perhaps reflecting differential kinetics in mRNA translation or protein degradation at baseline versus neurohormonal activation. Nevertheless, aldosterone induced MAP4 protein expression in si-Scramble NRVMs but not in si-Wipi1 NRVMs, suggesting that the correlation between Wipi1 and Map4 transcript expression is biologically significant. MAP4 is involved in microtubule stabilization $(48,49)$, myogenesis (50), myocyte metabolism (51), and inhibition of microtubule-based mRNA active transport (52). Overexpression of MAP4 in isolated cardiac myocytes and transgenic mice has been shown to cause cardiac myocyte contractile dysfunction (53). Our findings suggest that WIPI1 might regulate Map4 transcription itself and, in doing so, could theoretically affect MAP4-mediated cellular processes.

Our identification of HSPB6 as a driver of RVF is consistent with current understanding of HSPB6 but also presents potentially new insights. In our in vitro and in vivo RVF models, HSPB6 was upregulated but its phosphorylation was not. Phosphorylated HSPB6 plays important roles in cardiac contractile function (54-56), canonical autophagy $(30,57)$, and cardioprotection $(30,58)$. Increased cardiac expression of phosphorylated HSPB6 has been reported in the LV of advanced-HFrEF patients (30), suggesting excessive canonical autophagy in the failing LV. Our finding of increased phosphorylation of HSPB6 Ser16 in the failing LV of TAC mice is consistent with these human data. Whether nonphosphorylated HSPB6 has distinct pathophysiological actions or reflects deficiencies in processes otherwise mediated by phosphorylated HSPB6 remains unclear. Our findings suggest differences between the failing RV and the failing LV with regards to HSPB6 signaling and autophagy pathways.

Above all, our study stands out for its unbiased, comprehensive approach to identifying molecular pathophysiological signaling specific to the failing RV. Prior transcriptomic analyses of animal models and human tissue have relied on differential-expression and pathway analyses without subsequent experimental and mechanistic validation studies of proposed molecular signatures of RVF (13-20). Although single-gene and differential-expression analyses are powerful tools, there are a number of advantages with WGCNA (59). As a module-based approach, WGCNA is well suited to analyze large data sets and to take a more global view. Moreover, by examining the interaction patterns between genes to identify gene modules (networks), WGCNA filters results to a meaningful subset of the total expression data in an unsupervised, unbiased manner. 
WGCNA is also able to utilize and incorporate subtle shifts in gene expression, making it better able to elucidate true changes in samples compared with differential expression approaches. By correlating these modules to hemodynamic indices of RVF, we discovered a robust, biologically significant and interesting gene network. With WGCNA, we could leverage betweenness centrality to identify important actors in the alteration of a phenotype, whereas such an analysis is impossible in a list of differentially expressed genes. Further investigation of intramodular connectivity between genes allowed us to identify key genetic drivers or hubs that could be experimentally validated, targeted for therapeutics, or used as novel biomarkers. Additionally, WGCNA can link novel with known genes, thereby assisting in the identification of potential functions and biological processes of novel genes. For example, WGCNA has been instrumental in identifying genetic programs critical to embryonic development (60) and cardiac myocyte differentiation (61). Finally, because WGCNA is expression rather than interaction based, it is better able to identify large, high-impact modules driven by changes due to transcription factors and other global signaling processes as compared with an interaction-based network that excels at the exact recreation of already known pathways.

Limitations. Despite its robustness for understanding global patterns that underlie phenotypic traits WGCNA does have some limitations. Firstly, as a statistically driven method, WGCNA may link genes that are not involved in the exact same molecular pathway. Instead, linked genes may represent related but noninteracting members of 2 parallel processes. Thus, experimental validation is absolutely necessary. Secondly, the WGCNA algorithm may distort the true relationships between genes and phenotypes through the use of the soft-thresholding algorithm. The soft-thresholding algorithm alters expressed genes by raising them to an algorithm-guided power and uses only the first principle component of a module, which may represent only a small fraction of the total variance of a module, as a proxy measure for correlation between a phenotype and the entire module. Our first principle component accounted for $77 \%$ of the total variance (Supplemental Figure 6), suggesting that these concerns do not apply to our data. Thirdly, the algorithm used by Cytoscape to generate betweenness centralities is incapable of working with weighted edges. A weighted betweenness centrality approach may be able to more accurately identify hub genes. Lastly, because our modules are derived computationally, hubs that physically interact with many genes but which do not affect their expression will not be observed in our results. Despite these limitations, WGCNA and module-phenotype analyses still offer biologically significant insights that simply cannot be afforded by single-gene and differential-expression analyses. Importantly, these limitations of WGCNA can be addressed through experimental validation and testing, as we have done.

In vitro modeling of RVF is particularly challenging compared with that of LVF. Morphologic and physiologic differences between the RV and LV as well as those between the pulmonary and systemic vasculatures profoundly magnify the pathophysiologic role of increased arterial elastance (decreased pulmonary vascular compliance) in RVF (62). As suggested by our mouse model, chronicity of both pressure overload and neurohormonal activation are likely important determinants of WIPI1 expression. Thus, limitations inherent to in vitro cell culture models might explain why Wipi1 transcript and protein expression were upregulated in the failing RV of PAB mice but not in aldosterone-stimulated NRVMs. Despite this discrepancy, our in vitro studies with si-Wipil still revealed a significant functional role of WIPI1 in mediating aldosterone-induced mitochondrial superoxide levels. Importantly, others have already demonstrated that hyperaldosteronism is associated with RVF in large animal models (23) and patients (26). Moreover, mitochondrial ROS play a significant role in the pathophysiology of RVF but not that of LVF (63).

Our experimental data regarding the cardioprotective effect of silencing Wipi1 are limited to in vitro studies at this time. Silencing Wipil had the greatest effects on aldosterone-induced mitochondrial superoxide signaling and noncanonical autophagy but only a small impact on the fetal gene program. This highlights the limitation of using the fetal gene program as a surrogate outcome for RVF, rather than necessarily a limitation of the therapeutic potential of targeting Wipil. Given the known pathophysiological roles of aldosterone and mitochondrial ROS in RVF, the substantial blunting of aldosterone-induced mitochondrial superoxide levels and mitochondrial protein oxidation by si-Wipi1 is particularly significant. In vivo studies are needed to confirm the therapeutic potential of silencing or inhibiting Wipi1 in RVF.

Conclusions. In summary, our data demonstrate what we believe are novel roles for WIPI1 in regulating mitochondrial oxidative stress signaling, noncanonical autophagy, and MAP4 transcription in RVF. Silencing Wipi1 in an in vitro model of RVF-associated neurohormone activation decreased mitochondrial superoxide levels and mitochondrial protein oxidation, dampened excessive noncanonical autophagy, and reduced Map4 mRNA expression. Given the significance of RV dysfunction and RVF and the absence of 
RV-targeted therapies, further investigation into how WIPI1 mediates these processes is warranted and may lead to innovative therapies for RVF.

\section{Methods}

Study design. The goal of this study was to identify unique genetic determinants of RVF that might be targeted for the development of novel RVF-specific therapy. Towards this end, we leveraged transcriptomic data from human ventricular tissue of advanced-HF patients with versus without hemodynamically significant RVF as well as that from NF donors ( $n=5$ patients per group). Using WGCNA, module-trait analysis, and subsequent gene-phenotype correlations, we identified genes likely to mediate RVF. We experimentally validated some of the candidate RVF-associated genes in mouse models of pressure overload-induced RVF versus LVF. We subsequently focused our attention on the genetic hub WIPI1, which was upregulated in the failing RV of HF patients and mouse models and also correlated with other identified RVF-associated genetic drivers. To elucidate possible pathophysiological mechanisms of WIPI1 and test its potential as a therapeutic target, we performed in vitro-isolated cardiac myocyte cell culture studies, in which cells were subjected to neurohormonal activation associated with RVF, namely aldosterone activation. We silenced Wipil in this in vitro model and assessed the effect on autophagy, mitochondrial superoxide levels, and the fetal gene program associated with HF. Additional details can be found in the supplemental material.

Human ventricular tissue samples. Human ventricular myocardium was obtained from end-stage ischemic cardiomyopathic hearts explanted at the time of cardiac transplantation, as previously described (64). NF donor hearts that were unsuitable for transplantation were used as controls.

RNA-Seq. End-stage ischemic cardiomyopathic hearts were selected for RNA sequencing (RNA-Seq) based on patients' invasive hemodynamic parameters prior to transplantation and the absence of LV-assist device as a bridge to transplantation. The LVF cohort, defined as those without hemodynamic evidence of RVF, were selected based on RA less than $8 \mathrm{mmHg}$ and RA/PCWP less than 0.5. The BiV-HF cohort, defined as those with hemodynamic evidence of RVF and thereby biventricular HF, were selected based on $\mathrm{RA}$ greater than $15 \mathrm{mmHg}$ and RA/PCWP greater than 0.62 . These hemodynamic criteria for RVF were based on prior studies establishing cutoff values for RA (65) and RA/PCWP (2) in advanced-HF patients. Only matched LV and RV tissues (from the same patient) were used. RNA extraction was performed with a Total RNA Purification Plus Micro Kit (Norgen Biotek). RNA-Seq was performed by LC Sciences with the Illumina platform. The RNA-Seq data from this publication have been submitted to the NCBI's Gene Expression Omnibus (GEO) database (accession number GSE120852).

WGCNA. Networks were generated as previously described (22) using the parameters provided online (http://labs.genetics.ucla.edu/horvath/htdocs/CoexpressionNetwork/Rpackages/WGCNA/Tutorials/). The WGCNA method was implemented in the freely available WGCNA R package (22).

Module selection and enrichment. Eigengenes of the RV-only modules were correlated to RVF hemodynamic indices, and modules with significant correlations $(P<0.05)$ were further filtered to determine which RV modules were not preserved or weakly preserved in the combined network. This RV-specific, RVF-associated module was subsequently used to identify hubs and drivers. GeneAnalytics was used to identify enriched biological categories in the genes of our modules of interest (66). Significance was determined by a Benjamini-Hochberg-corrected binomial test $P<0.05$.

Hubs. Betweenness centrality was calculated for each transcript using the NetworkAnalyzer tool in Cytoscape (67). Transcripts with significant betweenness centralities (Hubs) have increased importance to overall modular structure. Significance was calculated by bootstrapping 100,000 networks with the same number of nodes and preserved degree structure using the degree.sequence.game function from the $\mathrm{R}$ package igraph (68) and an overall significance threshold (0.00029) determined by Bonferroni's correction.

Drivers and repressors. Drivers and repressors are genes connected to the rest of the module that respectively show strong independent positive or negative correlation with RVF hemodynamic indices. Genes with low betweenness centralities (lowest quartile) were removed. Genes significantly correlated to RVF hemodynamic indices were ranked based on $P$ value. Potential candidate drivers and repressors were selected based on significant correlations, betweenness centrality, and previously validated expression in human cardiac tissue (69).

Module visualization. Module visualization was performed using Cytoscape 3.4 (70). Node size reflected that node's betweenness centrality; node color reflected the transcript's correlation (direction and strength) to a composite RVF phenotype index averaging the correlation values of each transcript with RA, PASP, and MAP/ $\mathrm{RA}$. The negative of the MAP/RA correlation value was used in this averaged index because MAP/RA is 
inversely related to RVF. Hence, drivers have positive correlations to the RVF index, and repressors have negative correlations. Green node color indicates at least modest negative $\left(\mathrm{R}^{2}<-0.4\right)$, yellow indicates minimal $(-0.4$ $\left.\leq \mathrm{R}^{2} \leq 0.4\right)$, and red indicates at least modest positive $\left(\mathrm{R}^{2}>0.4\right)$ phenotypic correlations. Module layout was determined via the "edge-weighted spring embedded" layout algorithm using the correlation strength between individual gene expressions as the edge weights. Edges with $\mathrm{R}<0.88$ were removed to aid visualization.

Heatmaps. Heatmaps were generated using the heatmap. 2 function from the R package gplots (71).

RNA isolation and quantitative RT-PCR. Total RNA was extracted from animal tissues and cells using the Tissue RNA Purification Kit (Norgen Biotek) and TRIzol reagent (Invitrogen), respectively; quantified using a NanoDrop-2000c (Thermo Fisher Scientific); and reverse transcribed with iScript Supermix (BioRad). RT-qPCR was performed in triplicate using iTac Universal SYBR Green Supermix (Bio-Rad) and the CFX96 Touch Detection System (Bio-Rad). Primer sequences are listed in Supplemental Table 4. Gene expression was normalized to Rps 13 , and relative mRNA expression was quantified using the $2^{-\Delta \Delta C t}$ method.

Protein isolation and Western blot analysis. Total protein was prepared from tissues and cells by homogenization in T-PER lysis buffer (Thermo Fisher Scientific) supplemented with the Halt Protease and Phosphatase Inhibitor Cocktail (Thermo Fisher Scientific) and quantified using BCA assay (Pierce). Protein samples were resolved in NuPAGE 4\%-12\% Bis-Tris gradient gels (Invitrogen) and transferred to $0.2-\mu \mathrm{m}$ nitrocellulose membranes (Bio-Rad). Membranes were stained with the LI-COR Revert Total Protein Stain kit and analyzed. Membranes were subsequently blocked, incubated with primary antibodies at $4^{\circ} \mathrm{C}$ overnight, washed, and then incubated with the appropriate LI-COR secondary antibody. The ODYSSEY-Classic infrared imaging system (LI-COR) was used for quantitation. Target protein expression was normalized to total protein. Antibodies are listed in Supplemental Table 5.

Animal experiments. PAB, TAC, and sham surgeries were performed as previously described $(72,73)$ with slight modifications.

Echocardiography. Mice were anesthetized with 1\%-2\% inhalational isoflurane and transthoracic echocardiography was performed using a 18 - to $38-\mathrm{MHz}$ linear-array transducer probe with a digital ultrasound system (Vevo 2100 Image System, VisualSonics). Vevo LAB 3.0 ultrasound analysis software (Fujifilm, VisualSonics) was used to measure and analyze images.

Hemodynamic assessment of RV function. A subset of 6- and 9-week PAB/Sham mice underwent terminal hemodynamic assessment, as previously described (74) but modified for the RV. RV cardiac catheterization was performed using a closed-chest method whereby a Millar PVR-1035 (Millar Instruments) mouse conductance catheter was inserted into the right external jugular vein and advanced into the RV. Upon hemodynamic stability, steady-state baseline data were collected by the conductance catheter coupled to a Millar MPVS Ultra and PowerLab 16/35 data acquisition system (AD Instruments). RV pressure and volume waveforms were recorded simultaneously and analyzed over a minimum of 10 consecutive cardiac cycles using LabChart 8 software (AD Instruments).

Morphometric analysis. At experimental endpoints of 3, 6, or 9 weeks following surgery, mice were euthanized and heparinized, and ventricles, lungs, and liver were removed and weighed. Tissue weights were normalized to tibia length (TL) to assess pulmonary edema (lung weight/TL), hepatic congestion (liver weight/TL), and LV hypertrophy (LV/TL).

Isolation and primary culture of NRVMs. Pregnant female Sprague-Dawley rats were purchased from Charles River Laboratories. Neonatal Sprague-Dawley rats were euthanized by decapitation within the first 24 hours after birth and beating hearts were collected for digestion isolation of ventricular myocytes and single-layer cell culture as previously described (75) with modifications.

siRNA transfection of NRVMs and in vitro model of neurohormone activation associated with $R V F$. At 24 hours following isolation, NRVMs were transfected with $10 \mathrm{nM}$ nontargeting siRNA (si-Scramble) or Wipil-targeting siRNA (si-Wipi1) using Dharmafect 1 transfection reagent (Dharmacon). All siRNAs were ON-TARGETplus SMARTpool siRNAs (Dharmacon). Following 24-hour transfection, cells were serum starved in serum-free DMEM/F12 supplemented with penicillin (100 units $/ \mathrm{ml})$ and streptomycin $(100 \mu \mathrm{g} / \mathrm{ml})$ for 12 hours and then incubated with aldosterone $(1 \mu \mathrm{M})$ in serum-free DMEM/F12 culture medium for 48 hours at $37^{\circ} \mathrm{C}, 5 \% \mathrm{CO}_{2}$. Serum-free DMEM/F12 culture medium without any aldosterone was used as a comparative control for neurohormone activation. For autophagy studies, transfected NRVMs were treated with either BafA $(100 \mathrm{nM})$ or CQ $(100 \mu \mathrm{M})$ for 1 hour prior to harvesting (33) in complete MEM medium.

MTT assay of cell viability. To assess NRVM viability, the Vybrant MTT cell proliferation assay kit was used (Molecular Probes) according to the manufacturer's protocol. 
MitoSOX Red analysis. Mitochondrial superoxide level was monitored with the MitoSOX Red mitochondrial superoxide indicator (Molecular Probes) for live-cell imaging, according to the manufacturer's instructions. Cells were imaged with a DMI8 fluorescence microscope (Leica) using a red fluorescence filter (excitation/emission 510/580 nm). Red fluorescent signal was measured with ImageJ software (NIH) and normalized to the brightfield signal.

Redox Western blots. In NRVMs, the redox state of the mitochondrial proteins CYPD and TRX2 was estimated by redox Western blot as previously described (76). Briefly, NRVMs were transfected with si-WipiI or si-Scramble and incubated at $37^{\circ} \mathrm{C}$ under the following conditions: (i) nontreated control (18 hours); (ii) $N$-acetyl cysteine ( $2 \mathrm{mM}, 18$ hours); (iii) $\mathrm{H}_{2} \mathrm{O}_{2}(0.1 \mathrm{mM}, 18$ hours); and (iv) aldosterone ( $1 \mu \mathrm{M}, 48$ hours). Cells were harvested and incubated in urea lysis buffer $(8 \mathrm{M}$ urea, $50 \mathrm{mM}$ Tris $/ \mathrm{HCl}[\mathrm{pH} 8.3]$ and $1 \mathrm{mM}$ EDTA) containing $20 \mathrm{mM}$ iodoacetamide (IAM) to alkylate protein thiol groups. Protein was then cold-acetone precipitated from the lysate supernatant, washed, then resuspended and incubated in urea lysis buffer containing $5 \mathrm{mM}$ DTT to reduce the oxidized protein thiol groups. Subsequent incubation in $100 \mathrm{mM}$ iodoacetic acid (IAA) in urea/DTT lysis buffer (final IAA concentration $50 \mathrm{mM}$ ) was performed to alkylate DTT-reduced thiol groups. Alkylation reactions were performed in the dark. Following the serial IAM/IAA alkylation, samples were subjected to urea-PAGE electrophoresis ( $7 \mathrm{M}$ urea and $7.5 \%$ acrylamide) in nonreducing conditions and immunoblotted for CYPD and TRX2.

Statistics. Statistical analyses of mouse and NRVM experiments were carried out using GraphPad Prism 8.0. Unless otherwise specified, data are expressed as mean \pm SEM. Means between 2 groups were compared using unpaired, 2-tailed Student's $t$ test, unless otherwise specified. For redox Western blots, unpaired, 1-tailed Student's $t$ test was used to determine whether the decrease in oxidized proteins seen with silencing Wipil was statistically significant. Here, we sought to detect change in just one direction. For multiple comparisons, 1- or 2-way ANOVAs were performed, followed by Tukey's multiple-comparison tests. Statistical significance was defined as $P<0.05$. For the assessment of RNA-Seq data of each of the candidate WGCNA-identified RVF-associated genetic hub, drivers, and repressors, statistical significance was defined as $P<0.10$, given the limited sample size, non-normal data distribution, and use of human tissue analysis as a discovery rather than validation approach.

Study approval. Patient consent, sample collection and preparation, and clinical data collection were performed according to a human subject research protocol approved by the IRB of the Lewis Katz School of Medicine at Temple University (PI: Tsai). Animal experiments were conducted in strict accordance with the recommendations in the Guide for the Care and Use of Laboratory Animal of the NIH. All animal protocols were approved by the IACUC of Columbia University.

\section{Author contributions}

CT designed, performed, and analyzed all NRVM studies, conducted mouse research, contributed to manuscript writing, and created figures. CDR performed the WGCNA and computational biology analysis, contributed to manuscript writing, and created figures. PEB supported NRVM studies and mouse research and their associated data analyses. GJL, JC, and KL supported the mouse research and associated data analysis. WD performed and analyzed mouse echocardiograms, morphometrics, and invasive hemodynamic measurements and contributed to manuscript writing and figure creation. YW provided critical revisions to the manuscript. EJT conceived the project, designed the research, performed mouse echocardiograms, analyzed data, wrote the manuscript, and contributed to figure creation.

\section{Acknowledgments}

We thank Qing Li (Columbia University) for mouse surgical support and Haejung Chung (Columbia University) for technical support. This work was supported in part by American Heart Association grant 15POST25310006 to CDR; and American College of Cardiology Presidential Career Development Award and NIH grants K08HL109159, R03HL133706, and R01HL138528 to EJT.

Address correspondence to: Emily J. Tsai, 630 W. 168th Street, P\&S 8-510, New York, New York 10032, USA. Phone: 212.305.3409; Email: emily.tsai@columbia.edu.

EJT's present address: Columbia University Vagelos College of Physicians and Surgeons, New York, New York, USA. 
1. Cenkerova K, Dubrava J, Pokorna V, Kaluzay J, Jurkovicova O. Right ventricular systolic dysfunction and its prognostic value in heart failure with preserved ejection fraction. Acta Cardiol. 2015;70(4):387-393.

2. Drazner $\mathrm{MH}$, et al. Relationship of right- to left-sided ventricular filling pressures in advanced heart failure: insights from the ESCAPE trial. Circ Heart Fail. 2013;6(2):264-270.

3. Gerges M, et al. Pulmonary hypertension in heart failure. Epidemiology, right ventricular function, and survival. Am J Respir Crit Care Med. 2015;192(10):1234-1246.

4. Ghio S, et al. Prognostic relevance of a non-invasive evaluation of right ventricular function and pulmonary artery pressure in patients with chronic heart failure. Eur J Heart Fail. 2013;15(4):408-414.

5. Gulati A, et al. The prevalence and prognostic significance of right ventricular systolic dysfunction in nonischemic dilated cardiomyopathy. Circulation. 2013;128(15):1623-1633.

6. Peters A, Caroline M, Zhao H, Baldwin MR, Forfia PR, Tsai EJ. Initial right ventricular dysfunction severity identifies severe peripartum cardiomyopathy phenotype with worse early and overall outcomes: A 24-year cohort study. J Am Heart Assoc. 2018;7(9):null.

7. Forfia PR, et al. Tricuspid annular displacement predicts survival in pulmonary hypertension. Am J Respir Crit Care Med. 2006;174(9):1034-1041.

8. Haddad F, et al. Right heart score for predicting outcome in idiopathic, familial, or drug- and toxin-associated pulmonary arterial hypertension. JACC Cardiovasc Imaging. 2015;8(6):627-638.

9. van de Veerdonk MC, et al. Progressive right ventricular dysfunction in patients with pulmonary arterial hypertension responding to therapy. J Am Coll Cardiol. 2011;58(24):2511-2519.

10. Mohammed SF, et al. Right ventricular function in heart failure with preserved ejection fraction: a community-based study. Circulation. 2014;130(25):2310-2320.

11. Puwanant S, Priester TC, Mookadam F, Bruce CJ, Redfield MM, Chandrasekaran K. Right ventricular function in patients with preserved and reduced ejection fraction heart failure. Eur J Echocardiogr. 2009;10(6):733-737.

12. Yancy CW, et al. 2013 ACCF/AHA guideline for the management of heart failure: executive summary: a report of the American College of Cardiology Foundation/American Heart Association Task Force on practice guidelines. Circulation. 2013;128(16):1810-1852.

13. Drake JI, et al. Molecular signature of a right heart failure program in chronic severe pulmonary hypertension. Am J Respir Cell Mol Biol. 2011;45(6):1239-1247.

14. Gao Z, et al. Transcriptomic profiling of the canine tachycardia-induced heart failure model: global comparison to human and murine heart failure. J Mol Cell Cardiol. 2006;40(1):76-86.

15. Potus F, Hindmarch CCT, Dunham-Snary KJ, Stafford J, Archer SL. Transcriptomic signature of right ventricular failure in experimental pulmonary arterial hypertension: deep sequencing demonstrates mitochondrial, fibrotic, inflammatory and angiogenic abnormalities. Int J Mol Sci. 2018;19(9):null.

16. Reddy S, et al. Physiologic and molecular characterization of a murine model of right ventricular volume overload. Am J Physiol Heart Circ Physiol. 2013;304(10):H1314-H1327.

17. Urashima T, et al. Molecular and physiological characterization of RV remodeling in a murine model of pulmonary stenosis. Am J Physiol Heart Circ Physiol. 2008;295(3):H1351-H1368.

18. di Salvo TG, Yang KC, Brittain E, Absi T, Maltais S, Hemnes A. Right ventricular myocardial biomarkers in human heart failure. J Card Fail. 2015;21(5):398-411.

19. Di Salvo TG, et al. Right ventricular long noncoding RNA expression in human heart failure. Pulm Circ. 2015;5(1):135-161.

20. Williams JL, et al. Defining the molecular signatures of human right heart failure. Life Sci. 2018;196:118-126.

21. Khatri P, Sirota M, Butte AJ. Ten years of pathway analysis: current approaches and outstanding challenges. PLoS Comput Biol. 2012;8(2):e1002375.

22. Langfelder P, Horvath S. WGCNA: an R package for weighted correlation network analysis. BMC Bioinformatics. 2008;9:559.

23. Aguero J, et al. Characterization of right ventricular remodeling and failure in a chronic pulmonary hypertension model. Am $J$ Physiol Heart Circ Physiol. 2014;307(8):H1204-H1215.

24. Gregori $\mathrm{M}$, et al. Abnormal regulation of renin angiotensin aldosterone system is associated with right ventricular dysfunction in hypertension. Can J Cardiol. 2014;30(2):188-194.

25. Safdar Z, et al. Circulating aldosterone levels and disease severity in pulmonary arterial hypertension. J Pulm Respir Med. 2015;5(5):null.

26. Maron BA, Opotowsky AR, Landzberg MJ, Loscalzo J, Waxman AB, Leopold JA. Plasma aldosterone levels are elevated in patients with pulmonary arterial hypertension in the absence of left ventricular heart failure: a pilot study. Eur J Heart Fail. 2013;15(3):277-283.

27. Mleczak A, Millar S, Tooze SA, Olson MF, Chan EY. Regulation of autophagosome formation by Rho kinase. Cell Signal. 2013;25(1):1-11.

28. Tsuyuki S, et al. Detection of WIPI1 mRNA as an indicator of autophagosome formation. Autophagy. 2014;10(3):497-513.

29. Tanida I, Ueno T, Kominami E. LC3 and Autophagy. Methods Mol Biol. 2008;445:77-88.

30. Qian J, et al. Blockade of Hsp20 phosphorylation exacerbates cardiac ischemia/reperfusion injury by suppressed autophagy and increased cell death. Circ Res. 2009;105(12):1223-1231.

31. Codogno P, Mehrpour M, Proikas-Cezanne T. Canonical and non-canonical autophagy: variations on a common theme of self-eating? Nat Rev Mol Cell Biol. 2011;13(1):7-12.

32. Lazarou M, et al. The ubiquitin kinase PINK1 recruits autophagy receptors to induce mitophagy. Nature. 2015;524(7565):309-314.

33. Florey O, Gammoh N, Kim SE, Jiang X, Overholtzer M. V-ATPase and osmotic imbalances activate endolysosomal LC3 lipidation. Autophagy. 2015;11(1):88-99.

34. Martinez-Martin N, et al. A switch from canonical to noncanonical autophagy shapes B cell responses. Science. 2017;355(6325):641-647.

35. Dai DF, et al. Mitochondrial oxidative stress mediates angiotensin II-induced cardiac hypertrophy and Galphaq overexpression-induced heart failure. Circ Res. 2011;108(7):837-846. 
36. Lee J, Giordano S, Zhang J. Autophagy, mitochondria and oxidative stress: cross-talk and redox signalling. Biochem J. 2012;441(2):523-540.

37. Proikas-Cezanne T, Takacs Z, Dönnes P, Kohlbacher O. WIPI proteins: essential PtdIns3P effectors at the nascent autophagosome. J Cell Sci. 2015;128(2):207-217.

38. Hein S, et al. Progression from compensated hypertrophy to failure in the pressure-overloaded human heart: structural deterioration and compensatory mechanisms. Circulation. 2003;107(7):984-991.

39. Kostin S, et al. Myocytes die by multiple mechanisms in failing human hearts. Circ Res. 2003;92(7):715-724

40. Matsui Y, et al. Distinct roles of autophagy in the heart during ischemia and reperfusion: roles of AMP-activated protein kinase and Beclin 1 in mediating autophagy. Circ Res. 2007;100(6):914-922.

41. Nakai A, et al. The role of autophagy in cardiomyocytes in the basal state and in response to hemodynamic stress. Nat Med. 2007;13(5):619-624

42. Xu X, et al. Diminished autophagy limits cardiac injury in mouse models of type 1 diabetes. J Biol Chem. 2013;288(25):18077-18092.

43. $\mathrm{Zhu} \mathrm{H}$, et al. Cardiac autophagy is a maladaptive response to hemodynamic stress. J Clin Invest. 2007;117(7):1782-1793.

44. Shirakabe A, et al. Drp1-dependent mitochondrial autophagy plays a protective role against pressure overload-induced mitochondrial dysfunction and heart failure. Circulation. 2016;133(13):1249-1263.

45. Ucar A, et al. The miRNA-212/132 family regulates both cardiac hypertrophy and cardiomyocyte autophagy. Nat Commun. 2012;3:1078.

46. Wu P, et al. Myocardial upregulation of cathepsin D by ischemic heart disease promotes autophagic flux and protects against cardiac remodeling and heart failure. Circ Heart Fail. 2017;10(7):null.

47. Schlüter KD, Kutsche HS, Hirschhäuser C, Schreckenberg R, Schulz R. Review on chamber-specific differences in right and left heart reactive oxygen species handling. Front Physiol. 2018;9:1799.

48. Cheng G, et al. Basis for MAP4 dephosphorylation-related microtubule network densification in pressure overload cardiac hypertrophy. J Biol Chem. 2010;285(49):38125-38140.

49. Fassett JT, et al. AMPK attenuates microtubule proliferation in cardiac hypertrophy. Am J Physiol Heart Circ Physiol. 2013;304(5):H749-H758.

50. Mogessie B, Roth D, Rahil Z, Straube A. A novel isoform of MAP4 organises the paraxial microtubule array required for muscle cell differentiation. Elife. 2015;4:e05697.

51. Teng M, et al. Microtubular stability affects cardiomyocyte glycolysis by HIF-1alpha expression and endonuclear aggregation during early stages of hypoxia. Am J Physiol Heart Circ Physiol. 2010;298(6):H1919-H1931.

52. Scholz D, et al. Microtubule-associated protein-4 (MAP-4) inhibits microtubule-dependent distribution of mRNA in isolated neonatal cardiocytes. Cardiovasc Res. 2006;71(3):506-516.

53. Takahashi M, et al. Phenotypic consequences of beta1-tubulin expression and MAP4 decoration of microtubules in adult cardiocytes. Am J Physiol Heart Circ Physiol. 2003;285(5):H2072-H2083.

54. Chu G, et al. Phosphoproteome analysis of cardiomyocytes subjected to beta-adrenergic stimulation: identification and charac terization of a cardiac heat shock protein p20. Circ Res. 2004;94(2):184-193.

55. Pipkin W, Johnson JA, Creazzo TL, Burch J, Komalavilas P, Brophy C. Localization, macromolecular associations, and function of the small heat shock-related protein HSP20 in rat heart. Circulation. 2003;107(3):469-476.

56. Wang X, et al. Overexpression of Hsp20 prevents endotoxin-induced myocardial dysfunction and apoptosis via inhibition of NF-kappaB activation. J Mol Cell Cardiol. 2009;47(3):382-390.

57. Liu GS, et al. Regulation of BECN1-mediated autophagy by HSPB6: Insights from a human HSPB6. Autophagy. 2018;14(1):80-97.

58. Fan GC, et al. Novel cardioprotective role of a small heat-shock protein, Hsp20, against ischemia/reperfusion injury. Circulation. 2005;111(14):1792-1799.

59. Horvath S, et al. Analysis of oncogenic signaling networks in glioblastoma identifies ASPM as a molecular target. Proc Natl Acad Sci USA. 2006;103(46):17402-17407

60. Xue Z, et al. Genetic programs in human and mouse early embryos revealed by single-cell RNA sequencing. Nature. 2013;500(7464):593-597.

61. Liu Q, et al. Genome-wide temporal profiling of transcriptome and open chromatin of early cardiomyocyte differentiation derived from hiPSCs and hESCs. Circ Res. 2017;121(4):376-391.

62. Thenappan T, Prins KW, Pritzker MR, Scandurra J, Volmers K, Weir EK. The critical role of pulmonary arterial compliance in pulmonary hypertension. Ann Am Thorac Soc. 2016;13(2):276-284.

63. Redout EM, et al. Right-ventricular failure is associated with increased mitochondrial complex II activity and production of reactive oxygen species. Cardiovasc Res. 2007;75(4):770-781.

64. Dipla K, Mattiello JA, Jeevanandam V, Houser SR, Margulies KB. Myocyte recovery after mechanical circulatory support in humans with end-stage heart failure. Circulation. 1998;97(23):2316-2322.

65. Atluri P, et al. Predicting right ventricular failure in the modern, continuous flow left ventricular assist device era. Ann Thorac Surg. 2013;96(3):857-863.

66. Ben-Ari Fuchs S, et al. GeneAnalytics: an integrative gene set analysis tool for next generation sequencing, RNAseq and microarray data. OMICS. 2016;20(3):139-151.

67. Assenov Y, Ramírez F, Schelhorn SE, Lengauer T, Albrecht M. Computing topological parameters of biological networks. Bioinformatics. 2008;24(2):282-284.

68. Csardi G, Nepusz T. The igraph software package for complex network research. http://igraph.org/r/. Updated 2006. Accessed September 2017.

69. Fagerberg L, et al. Analysis of the human tissue-specific expression by genome-wide integration of transcriptomics and antibody-based proteomics. Mol Cell Proteomics. 2014;13(2):397-406.

70. Shannon P, et al. Cytoscape: a software environment for integrated models of biomolecular interaction networks. Genome Res. 2003;13(11):2498-2504.

71. Warnes GR, et al. gplots: Various R programming tools for plotting data. R Project. https://cran.r-project.org/package=gplots. Accessed April 30, 2019 
72. Rockman HA, et al. Segregation of atrial-specific and inducible expression of an atrial natriuretic factor transgene in an in vivo murine model of cardiac hypertrophy. Proc Natl Acad Sci USA. 1991;88(18):8277-8281.

73. Rockman HA, et al. Molecular and physiological alterations in murine ventricular dysfunction. Proc Natl Acad Sci USA. 1994;91(7):2694-2698.

74. Georgakopoulos D, et al. In vivo murine left ventricular pressure-volume relations by miniaturized conductance micromanometry. Am J Physiol. 1998;274(4):H1416-H1422.

75. Simpson P, Savion S. Differentiation of rat myocytes in single cell cultures with and without proliferating nonmyocardial cells Cross-striations, ultrastructure, and chronotropic response to isoproterenol. Circ Res. 1982;50(1):101-116.

76. Folda A, et al. Mitochondrial thioredoxin system as a modulator of cyclophilin D redox state. Sci Rep. 2016;6:23071. 